\title{
LIFTINGS OF DIAGRAMS OF SEMILATTICES BY DIAGRAMS OF DIMENSION GROUPS
}

\author{
JIŘÍ TŮMA AND FRIEDRICH WEHRUNG
}

\section{INTRODUCTION}

There are various ways to obtain distributive semilattices from other mathematical objects. Two of them are the following; we refer to Section 1 for more precise definitions. A dimension group is a directed, unperforated partially ordered abelian group with the interpolation property, see also K. R. Goodearl [6]. With a dimension group $G$ we can associate its semilattice of compact ( = finitely generated) ideals $\operatorname{Id}_{\mathrm{c}} G$. Because of the interpolation property the positive cone $G^{+}$has the refinement property, thus the compact ideal semilattice $\operatorname{Id}_{\mathrm{c}} G$ is distributive. A ring $R$ is locally matricial over a field $K$, if it is isomorphic to a direct limit of finite products of full matricial rings $\mathcal{M}_{n}(K)$. If $R$ is locally matricial, or, more generally, regular (in von Neumann's sense), then its semilattice $\operatorname{Id}_{\mathrm{c}} R$ of compact ideals is distributive, see, for example, K. R. Goodearl [5].

These two different contexts are related as follows. With a locally matricial ring $R$, we can associate its (partially ordered) Grothendieck group $K_{0}(R)$. It turns out that $K_{0}(R)$ is a dimension group, and the following relation holds:

$$
\operatorname{Id}_{\mathrm{c}} R \cong \operatorname{Id}_{\mathrm{c}}\left(K_{0}(R)\right) \text {, }
$$

see [5. Corollary 15.21]. Therefore, the compact ideal semilattice of a locally matricial ring is the compact ideal lattice of a dimension group. By the results of K. R. Goodearl and D. E. Handelman [7, the converse holds for dimension groups of cardinality at most $\aleph_{1}$, but it fails for larger dimension groups, see F. Wehrung 17, 18. Nevertheless this makes it worthwhile to study the functor that with a dimension group $G$ associates its semilattice of compact ideals $\operatorname{Id}_{c} G$. For a survey paper on this and related issues we refer the reader to K. R. Goodearl and F. Wehrung 8 .

The central open problem that motivates the present paper is Problem 10.1 of $[\underline{8}$, that asks whether any distributive $\langle\vee, 0\rangle$-semilattice of cardinality at most $\aleph_{1}$ is isomorphic to the compact ideal semilattice of some dimension group. Let us call this the compact ideal representation problem. By [7, this is equivalent to asking whether any distributive $\langle\vee, 0\rangle$-semilattice of cardinality at most $\aleph_{1}$ is isomorphic to the compact ideal semilattice of some locally matricial ring. Although we do not solve this problem here, we settle related issues with some interest of their own,

2000 Mathematics Subject Classification. 06A12, 06C20, 06F20, 15A03, 15A24, 15A48, 16E20, 16E50, 19A49, 19K14.

Key words and phrases. Distributive semilattice, dimension group, locally matricial algebra, compact ideal, direct limit, flat, generic.

The authors were supported by the BARRANDE program and by the institutional grant CEZ:J13/98:113200007a. The first author was also partially supported by GA CR 201/99 and by GA UK 162/1999. 
and, hopefully, that will provide a stepping stone towards a solution for the general problem.

We first recall the answers to some related questions. As a corollary of the main result of G. M. Bergman [2] and a theorem of K. R. Goodearl [5], the following result is obtained, see also 8 for different proofs.

Theorem 0.1. Every countable distributive $\langle\vee, 0\rangle$-semilattice $S$ is isomorphic to $\mathrm{Id}_{\mathrm{c}} G$ for some countable dimension group $G$.

Another result of 8 is the following.

Theorem 0.2. Every distributive lattice $L$ with zero is isomorphic to $\operatorname{Id}_{\mathrm{c}} G$ for some dimension group $G$.

On the other hand, P. Růžička [14] constructs a distributive $\langle\vee, 0\rangle$-semilattice of cardinality $\aleph_{2}$ that is not isomorphic to $\operatorname{Id}_{\mathrm{c}} G$ for any dimension group $G$.

These results leave open the case of distributive $\langle\vee, 0\rangle$-semilattices of cardinality $\aleph_{1}$. By P. Pudlák 13, every distributive $\langle\vee, 0\rangle$-semilattice is isomorphic to the direct union of its finite distributive $\langle\vee, 0\rangle$-subsemilattices. A variant of this result, stating that every distributive $\langle\vee, 0\rangle$-semilattice is a direct limit of finite Boolean $\langle\vee, 0\rangle$-semilattices, is proved in $[8$. Thus it is natural to try an inductive approach to the compact ideal representation problem for distributive semilattices of cardinality $\aleph_{1}$ : find a simultaneous representation of a suitable limit system of finite Boolean semilattices that converges to a given distributive semilattice $S$ of cardinality $\aleph_{1}$. By A. P. Huhn [10], one can assume that the limit system is indexed by an infinite lattice in which every principal ideal is a finite dismantlable lattice.

After preliminary results, most notably about the existence of the so-called $\lambda$ generic maps in Sections 4 and 5 we prove in Section [6 our main results:

Theorem 6.4. Every finite dismantlable diagram of finite Boolean $\langle\vee, 0\rangle$-semilattices has a simultaneous representation with respect to the functor $\operatorname{Id}_{\mathrm{c}}$.

Theorem 17.1. For any countable distributive $\langle\vee, 0\rangle$-semilattice $S$ and any countable dimension vector space $H$, every $\langle\vee, 0\rangle$-homomorphism $\boldsymbol{f}: S \rightarrow \operatorname{Id}_{\mathrm{c}} H$ can be lifted by a positive homomorphism $f: G \rightarrow H$ for some dimension vector space $G$.

We also state consequences of Theorem 7.1 as well in ring theory as in lattice theory, see Corollaries 7.4 and 7.5

The remaining sections contain various counterexamples to other strategies for a positive solution of the compact ideal representation problem for distributive semilattices of cardinality $\aleph_{1}$.

\section{BASIC CONCEPTS}

For basic concepts about partially ordered abelian groups, we refer the reader to K. R. Goodearl 6]. We recall some of the definitions here. For a partially ordered abelian group $G$, we denote by $G^{+}=\{x \in G \mid 0 \leq x\}$ the positive cone of $G$, and we say that $G$ is directed, if $G=G^{+}+\left(-G^{+}\right)$. We put $G^{++}=G^{+} \backslash\{0\}$, and we note $\mathbb{N}=\mathbb{Z}^{++}$. A subgroup $H$ of $G$ is convex, if $0 \leq x \leq a$ implies that $x \in H$, for all $a \in H$ and $x \in G$. We say that $H$ is an ideal of $G$, if it is a directed, convex subgroup of $G$, and a compact ideal of $G$, if it is an ideal generated by a finite subset of $G$. 
As every ideal of $G$ is directed, any ideal (resp., compact ideal) of $G$ is generated (as an ideal) by a subset of $G^{+}$(resp., finite subset of $G^{+}$). In the latter case, one may replace a finite subset $\left\{a_{1}, \ldots, a_{n}\right\}$ of $G^{+}$by the singleton $\{a\}$, where $a=\sum_{i=1}^{n} a_{i}$, so the compact ideals of $G$ are exactly the subsets of $G$ of the form

$$
G(a)=\left\{x \in G \mid \exists n \in \mathbb{Z}^{+} \text {such that }-n a \leq x \leq n a\right\}, \quad \text { for } a \in G^{+} .
$$

An order-unit of $G$ is an element $a \in G^{+}$such that $G(a)=G$. We denote by $\operatorname{Id} G$ the lattice of ideals of $G$, and by $\operatorname{Id}_{\mathrm{c}} G$ the $\langle\vee, 0\rangle$-semilattice of compact ideals of $G$. Observe that $\operatorname{Id} G$ is an algebraic lattice and that $\operatorname{Id}_{c} G$ is its $\langle\vee, 0\rangle$-semilattice of compact elements, see G. Grätzer [9] for unexplained terminology. For elements $a, b \in G^{+}$, we write $a \propto b$, if there exists $n \in \mathbb{N}$ such that $a \leq n b$, and $a \asymp b$, if $a \propto b \propto a$. Hence

$$
\operatorname{Id}_{\mathrm{c}} G=\left\{G(a) \mid a \in G^{+}\right\},
$$

with containment and equality among the $G(a)$-s determined by

$$
G(a) \subseteq G(b) \text { iff } a \propto b \quad \text { and } \quad G(a)=G(b) \text { iff } a \asymp b,
$$

for all $a, b \in G^{+}$. Hence, $\operatorname{Id}_{\mathrm{c}} G$ is isomorphic to $\nabla\left(G^{+}\right)$, the maximal semilattice quotient of $G^{+}$, see $[$] .

The assignment $G \mapsto \operatorname{Id}_{c} G$ can be naturally extended to a functor, by defining, for a positive homomorphism (that is, an order-preserving group homomorphism) $f: G \rightarrow H$ of partially ordered abelian groups and a compact ideal $I$ of $G,\left(\operatorname{Id}_{\mathrm{c}} f\right)(I)$ as the compact ideal of $H$ generated by the image $f[I]$ of $I$ under $f$. Hence, $\left(\operatorname{Id}_{\mathrm{c}} f\right)(G(a))=H(f(a))$, for all $a \in G^{+}$, and it is an easy exercise to verify that the functor $\operatorname{Id}_{\mathrm{c}}$ thus defined preserves direct limits.

Most of the partially ordered abelian groups we shall deal with will be equipped with an additional structure of vector space, always over the field $\mathbb{Q}$ of rational numbers. Any partially ordered vector space $E$ has the additional property that $m x \geq 0$ implies that $x \geq 0$, for all $m \in \mathbb{N}$ and all $x \in E$, we say that $E$ is unperforated, see [6]. We observe that all group homomorphisms between vector spaces preserve the vector space structure.

A refinement monoid (see A. Tarski [15, F. Wehrung [16]) is a commutative monoid $M$ such that for any positive integers $m, n$ and elements $a_{0}, \ldots, a_{m-1}, b_{0}$, $\ldots, b_{n-1}$ of $M$ such that $\sum_{i<m} a_{i}=\sum_{j<n} b_{j}$, there are elements $c_{i, j}$ (for $i<m$ and $j<n)$ such that $a_{i}=\sum_{j<n} c_{i, j}$, for all $i<m$, and $b_{j}=\sum_{i<m} c_{i, j}$, for all $j<n$.

A partially ordered abelian group $G$ is an interpolation group (see [6]) if for all positive integers $m, n$ and elements $a_{0}, \ldots, a_{m-1}, b_{0}, \ldots, b_{n-1}$ of $G$ such that $a_{i} \leq b_{j}$, for all $i<m$ and $j<n$, there exists $x \in G$ such that $a_{i} \leq x \leq b_{j}$, for all $i<m$ and $j<n$. Equivalently, the positive cone $G^{+}$is a refinement monoid (see [6, Proposition 2.1]). In addition, we say that $G$ is a dimension group (see [6]) if $G$ is directed and unperforated. If $G$ is an interpolation group, then $\operatorname{Id}_{c} G$ is a refinement semilattice. Refinement semilattices are usually called distributive semilattices, see 9 .

We can prove right away the following very elementary lifting result:

Proposition 1.1. For every $\langle\vee, 0\rangle$-semilattice $S$, there exists a partially ordered vector space $E$ such that $\operatorname{Id}_{\mathrm{c}} E \cong S$.

Proof. We first observe that $S$ can be $\langle\vee, 0\rangle$-embedded into some powerset semilattice $\mathcal{P}(X)$, for a set $X$. For example, take $X=S$ and use the embedding 
that with any $s \in S$ associates $j(s)=\{x \in S \mid s \not \leq x\}$. We then let $F$ denote the vector space of all maps $f: X \rightarrow \mathbb{Q}$ with finite range. For any $f \in F$, we put $\operatorname{supp} f=\{x \in X \mid f(x) \neq 0\}$, and we put

$$
M=\{f \in F \mid f(x) \geq 0, \text { for all } x \in X \text { and } \operatorname{supp} f \in S\} .
$$

Then $M$ is an additive submonoid of $F$ (in particular, it is cancellative), $M \cap$ $(-M)=\{0\}$, and $M$ is closed under multiplication by nonnegative scalars. Hence $M$ is the positive cone of a structure of directed partially ordered vector space on $E=M+(-M)$. Let $\pi: M \rightarrow S$ be the map defined by the rule $\pi(f)=\operatorname{supp} f$, for all $f \in M$. Then $\pi(f) \leq \pi(g)$ (resp., $\pi(f)=\pi(g))$ iff $f \propto g$ (resp., $f \asymp g$ ) in $E$, for all $f, g \in M$, whence $\pi$ induces an isomorphism $\varphi$ from $\operatorname{Id}_{\mathrm{c}} E$ onto $S$ by the rule $\varphi(E(f))=\operatorname{supp} f$, for all $f \in M$.

Unfortunately, even if $S$ is distributive, the partially ordered vector space $E$ constructed above may not have interpolation.

Throughout the paper we shall often formulate our results in basic categorical language. Among the main categories we shall be working with are the following:

- The category $\mathcal{E}$ of pairs $A=\left(A_{0}, 1_{A}\right)$, where $A_{0}$ is a partially ordered vector space (that we will subsequently identify with $A$ ) and $1_{A}$ is an order-unit of $A_{0}$. For objects $A$ and $B$ of $\mathcal{E}$, a homomorphism, or positive homomorphism, from $A$ to $B$ is a positive homomorphism $f: A_{0} \rightarrow B_{0}$. Observe that we do not require that $f\left(1_{A}\right)=1_{B}$. We say that a homomorphism $f: A \rightarrow B$ is normalized, if $f\left(1_{A}\right)=1_{B}$. In particular, we will slightly abuse terminology by using isomorphisms only in the 'normalized' sense, that is, an isomorphism $f: A \rightarrow B$ satisfies the equality $f\left(1_{A}\right)=1_{B}$.

- The full subcategory $\mathcal{E}_{\mathrm{d}}$ of $\mathcal{E}$ consisting of all dimension vector spaces in $\mathcal{E}$.

— The category $\mathcal{S}$ of $\langle\vee, 0\rangle$-semilattices and $\langle\vee, 0\rangle$-homomorphisms.

- The full subcategory $\mathcal{S}_{\mathrm{fb}}$ of $\mathcal{S}$ whose objects are the finite Boolean semilattices.

In particular, $\operatorname{Id}_{\mathrm{c}}$ defines, by restriction, a functor from $\mathcal{E}$ to $\mathcal{S}$ that preserves direct limits.

Every partially ordered set $(P, \leq)$ can be viewed as a category with objects $x \in P$ and a unique morphism $\varepsilon_{x, y}: x \rightarrow y$ whenever $x \leq y$ in $P$. Let $\mathcal{E}^{\prime}$ be a subcategory of $\mathcal{E}$. We say that a diagram $\Phi:(P, \leq) \rightarrow \mathcal{S}$ of semilattices (i.e., a functor from $P$, viewed as a category, to $\mathcal{S}$ ) has a lifting, with respect to the functor $\operatorname{Id}_{c}$, to the category $\mathcal{E}^{\prime}$, if there is a diagram $\Psi:(P, \leq) \rightarrow \mathcal{E}^{\prime}$ such that the two functors $\Phi$ and $\operatorname{Id}_{\mathrm{c}} \circ \Psi$ are naturally equivalent, that is, if there are isomorphisms $\iota_{x}: \Phi(x) \rightarrow \operatorname{Id}_{\mathrm{c}}(\Psi(x))$, for $x \in P$, such that the following diagram commutes,

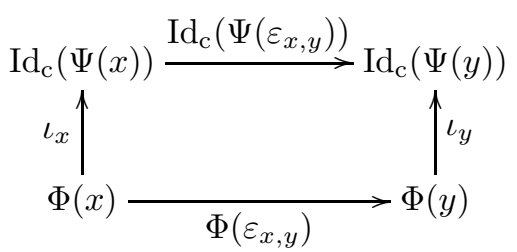

for all $x \leq y$ in $P$. We will usually omit the phrase with respect to $\mathrm{Id}_{\mathrm{c}}$, since lifts with respect to other functors will not be investigated.

For a set $I$, we denote by $\mathcal{P}(I)$ the powerset of $I$, and, for any $i \in I$, we put

$$
\mathcal{P}_{i}(I)=\{X \in \mathcal{P}(I) \mid i \in X\}, \quad \mathcal{P}_{i}^{*}(I)=\{X \in \mathcal{P}(I) \mid i \notin X\} .
$$




\section{Pseudo-Simplicial SPACES}

For a nonempty, finite set $X$, let $\mathbb{Q}_{X}$ denote the partially ordered vector space with underlying vector space $\mathbb{Q}^{X}$ and partial ordering defined by

$$
f \leq g \Leftrightarrow \text { either } f=g \text { or } f(x)<g(x) \text {, for all } x \in X,
$$

endowed with the canonical order-unit $1^{X}$, defined as the constant function on $X$ with value 1 . Then $\mathbb{Q}_{X}$ is a dimension vector space. It is simple, that is, $\operatorname{Id}_{\mathrm{c}} \mathbb{Q}_{X} \cong$ 2. We denote by $\left(1_{x}^{X}\right)_{x \in X}$ the canonical basis of $\mathbb{Q}^{X}$, so that $1^{X}=\sum_{x \in X} 1_{x}^{X}$. We observe that while $1^{X} \in\left(\mathbb{Q}_{X}\right)^{++}$, the vectors $1_{x}^{X}$, for $x \in X$, do not belong to $\left(\mathbb{Q}_{X}\right)^{++}$unless $X$ is a singleton. Nevertheless they keep a certain positivity character, captured by the following definition:

Definition 2.1. For a partially ordered abelian group $G$, we define a partial preordering $\leq{ }^{\text {arch }}$ on $G$ by the rule

$$
x \leq^{\text {arch }} y \Longleftrightarrow \exists u \in G \text { such that } n x \leq n y+u, \text { for all } n \in \mathbb{Z}^{+},
$$

for all $x, y \in G$. Then we define the partially preordered abelian group $G_{\text {arch }}=$ $\left(G, \leq^{\text {arch }}\right)$, the archimedean quotient of $G$.

In particular, for any finite set $X$, the archimedean quotient of $\mathbb{Q}_{X}$ is $\mathbb{Q}^{X}$, and $0<<^{\operatorname{arch}} 1_{x}^{X}$, for all $x \in X$.

Definition 2.2. An object $A$ of $\mathcal{E}$ is

(i) simple pseudo-simplicial, if $A \cong \mathbb{Q}_{X}$ for some nonempty finite set $X$,

(ii) pseudo-simplicial, if $A=\bigoplus_{i<n} A_{i}$ for some natural number $n$ and some simple pseudo-simplicial spaces $A_{i}$, for $i<n$.

The formula in (ii) above means that $A=\bigoplus_{i<n} A_{i}$ as vector spaces, $1_{A}=$ $\sum_{i<n} 1_{A_{i}}$, and the canonical bijection from $\prod_{i<n} A_{i}$ to $A$ is an isomorphism in $\mathcal{E}$ (that is, for the vector space structure as well as the - componentwise - partial order structure).

In particular, simplicial vector spaces, that is, spaces of the form $\mathbb{Q}^{n}$ with the positive cone $\left(\mathbb{Q}^{n}\right)^{+}$consisting of all vectors with non-negative coordinates, are particular cases of pseudo-simplicial spaces. Observe that for any pseudo-simplicial space $E$, the space $E_{\text {arch }}$ is simplicial.

For a simple pseudo-simplicial space $A$, the canonical basis of $A$ is defined as the set $T$ of minimal elements $t$ of $A_{\text {arch }}^{++}$such that $t \wedge\left(1_{A}-t\right)=0$, where $\wedge$ denotes the meet operation in the lattice-ordered group $A_{\text {arch }}$. Hence $T$ is a (finite) vector space basis of $A$ and the map from $\mathbb{Q}_{T}$ to $A$ that with any sequence $\left(x_{t}\right)_{t \in T}$ associates $\sum_{t \in T} x_{t} t$ is an isomorphism from $\mathbb{Q}_{T}$ onto $A$. It sends $1_{t}^{T}$ to $t$, for all $t \in T$, and $1^{T}$ to $1_{A}$.

We denote by $\mathcal{E}_{\mathrm{ps}}$ the full subcategory of $\mathcal{E}$ whose objects are the pseudo-simplicial spaces.

We state without proof the following lemma, that summarizes some elementary properties of pseudo-simplicial spaces.

Lemma 2.3. Let $m$ be a natural number, let $A_{0}, \ldots, A_{m-1}$ be simple pseudo-simplicial spaces. Put $A=\bigoplus_{i<m} A_{i}$, and identify $A_{i}$ with its canonical image in $A$, for all $i<m$. Then the following assertions hold.

(i) The ideals of $A$ are exactly the subsets of the form $\bigoplus_{i \in I} A_{i}$ for a subset $I$ of $\{0,1, \ldots, m-1\}$. 
(ii) The simple ideals of $A$ are exactly the $A_{i}$, for $i<m$.

(iii) $\operatorname{Id}_{\mathrm{c}} A \cong \mathbf{2}^{m}$, a finite Boolean semilattice.

For a partially ordered vector space $E$, the two binary relations $\propto$ and $\asymp$ on $E^{+}$ can be refined as follows. For a positive, rational number $\lambda$ and $a, b \in E^{+}$, we introduce the following notations:

$$
\begin{aligned}
a \propto_{\lambda} b, & \text { if } a \leq \lambda b ; \\
a \asymp_{\lambda} b, & \text { if } a \propto_{\lambda} b \text { and } b \propto_{\lambda} a ; \\
a \propto_{\lambda}^{\operatorname{arch}} b, & \text { if } a \propto_{\lambda+\varepsilon} b, \quad \text { for all rational } \varepsilon>0 ; \\
a \asymp_{\lambda}^{\operatorname{arch}} b, & \text { if } a \asymp_{\lambda+\varepsilon} b, \quad \text { for all rational } \varepsilon>0 .
\end{aligned}
$$

Hence $a \propto_{\lambda}^{\text {arch }} b$ (resp., $a \asymp_{\lambda}^{\operatorname{arch}} b$ ) in $E$ implies that $a \propto_{\lambda} b$ (resp., $a \asymp_{\lambda} b$ ) in $E_{\text {arch }}$; the converse holds if $E$ is simple.

\section{BASIC FACTS ABOUt REFINEMENT}

We start with an easy and useful lemma.

Lemma 3.1. Let $M$ be a refinement monoid, let $I$ and $T_{i}, i \in I$, be finite nonempty sets, let $a, a_{i, j}\left(\right.$ for $i \in I$ and $\left.j \in T_{i}\right)$ be elements of $M$ such that

$$
a=\sum_{j \in T_{i}} a_{i, j}, \quad \text { for all } i \in I \text {. }
$$

Then there are elements $x_{\varphi}\left(\right.$ for $\left.\varphi \in T=\prod_{i \in I} T_{i}\right)$ of $M$ such that

$$
a=\sum_{\varphi \in T} x_{\varphi} \quad \text { and } \quad a_{i, j}=\sum_{\substack{\varphi \in T \\ \varphi(i)=j}} x_{\varphi}, \quad \text { for all } \quad i \in I \quad \text { and } j \in T_{i} .
$$

Proof. An easy induction on the cardinality of $I$. There is nothing to prove if $|I|=1$. Now assume that the claim holds for any set $I$ of cardinality $n \geq 1$ and $I^{\prime}=I \cup\{k\} \neq I$. By the induction hypothesis on $I$ we get elements $x_{\varphi} \in M$ for $\varphi \in T$ such that

$$
a=\sum_{\varphi \in T} x_{\varphi} \quad \text { and } \quad a_{i, j}=\sum_{\substack{\varphi \in T \\ \varphi(i)=j}} x_{\varphi}, \quad \text { for all } \quad i \in I \quad \text { and } j \in T_{i} .
$$

Since also $a=\sum_{l \in T_{k}} a_{k, l}$ and $M$ is a refinement monoid, we get that there are elements $x_{\varphi, l} \in M$, for $\varphi \in T$ and $l \in T_{k}$, such that

$$
x_{\varphi}=\sum_{l \in T_{k}} x_{\varphi, l} \quad \text { and } \quad a_{k, l}=\sum_{\varphi \in T} x_{\varphi, l}
$$

for any $\varphi \in T$ and $l \in T_{k}$. Thus

$$
a=\sum_{l \in T_{k}} a_{k, l}=\sum_{l \in T_{k}} \sum_{\varphi \in T} x_{\varphi, l} .
$$

Since $T \times T_{k}=\prod_{i \in I \cup\{k\}} T_{i}$, the indices $(\varphi, l)$ are in one-to-one correspondence with functions $\psi \in T \times T_{k}$. Moreover, for every $i \in I$ and $j \in T_{i}$ we get

$$
a_{i, j}=\sum_{\substack{\varphi \in T \\ \varphi(i)=j}} x_{\varphi}=\sum_{\substack{\varphi \in T \\ \varphi(i)=j}} \sum_{l \in T_{k}} x_{\varphi, l}=\sum_{\substack{\psi \in T \times T_{k} \\ \psi(i)=j}} x_{\psi} .
$$


Finally, for every $l \in T_{k}$ we get

$$
a_{k, l}=\sum_{\varphi \in T} x_{\varphi, l}=\sum_{\substack{\psi \in T \times T_{k} \\ \psi(k)=l}} x_{\psi} .
$$

Lemma 3.2. Let $\lambda \geq 1$ be a rational number, let $E$ be a dimension vector space, let $I$ be a finite set, let $a_{i}($ for $i \in I)$ be elements of $E^{+}$. Then the following are equivalent:

(i) $a_{i} \asymp_{\lambda} a_{j}$, for all $i, j \in I$.

(ii) There are elements $b_{X}($ for $X \in \mathcal{P}(I))$ of $E^{+}$such that

$$
a_{i}=\sum_{X \in \mathcal{P}_{i}^{*}(I)} b_{X}+\lambda \cdot \sum_{X \in \mathcal{P}_{i}(I)} b_{X}, \quad \text { for all } i \in I .
$$

Proof. (ii) $\Rightarrow$ (i) is trivial.

Now assume that (i) holds. By applying interpolation to the system of inequalities $\lambda^{-1} a_{i} \leq a_{j}$, for all $i, j \in I$, we obtain $a \in E$ such that $\lambda^{-1} a_{i} \leq a \leq a_{j}$, for all $i, j \in I$. Hence, $a \leq a_{i} \leq \lambda a$, for all $i \in I$, so that there are $a_{i}^{\prime}, a_{i}^{\prime \prime} \in E^{+}$such that $a_{i}=a+a_{i}^{\prime}$ and $(\lambda-1) a=a_{i}^{\prime}+a_{i}^{\prime \prime}$, for all $i \in I$. By Lemma 3.1 applied to $M=E^{+}$ and $J=\{0,1\}$, there are elements $b_{X}$ of $E^{+}$, for $X \in \mathcal{P}(I)$, such that

$$
\begin{aligned}
a_{i}^{\prime}=(\lambda-1) \sum_{X \in \mathcal{P}_{i}(I)} b_{X}, \quad & a_{i}^{\prime \prime}=(\lambda-1) \sum_{X \in \mathcal{P}_{i}^{*}(I)} b_{X}, \quad \text { for all } i \in I ; \\
a & =\sum_{X \in \mathcal{P}(I)} b_{X} .
\end{aligned}
$$

Therefore, for all $i \in I$,

$$
\begin{aligned}
a_{i} & =\sum_{X \in \mathcal{P}(I)} b_{X}+(\lambda-1) \sum_{X \in \mathcal{P}_{i}(I)} b_{X} \\
& =\sum_{X \in \mathcal{P}_{i}^{*}(I)} b_{X}+\lambda \cdot \sum_{X \in \mathcal{P}_{i}(I)} b_{X} .
\end{aligned}
$$

\section{Flat AND GENERIC homomorphisms With SIMPle target}

Definition 4.1. Let $m$ be a natural number, let $A_{0}, \ldots, A_{m-1}, B$ be simple objects of $\mathcal{E}_{\mathrm{d}}$, let $f: \bigoplus_{i<m} A_{i} \rightarrow B$ be a positive homomorphism, let $\lambda \geq 1$ be a rational number. We say that $f$ is

(i) $\lambda$-flat, if $f\left(1_{A_{i}}\right) \asymp_{\lambda}^{\text {arch }} f\left(1_{A_{j}}\right)$, for all $i, j<m$ such that $f\left(1_{A_{i}}\right), f\left(1_{A_{j}}\right) \neq 0$.

(ii) $\lambda$-generic, if for any simple pseudo-simplicial space $C$ and for any $\lambda$-flat homomorphism $g: \bigoplus_{i<m} A_{i} \rightarrow C$ such that

$$
f\left[A_{i}\right] \neq\{0\} \Leftrightarrow g\left[A_{i}\right] \neq\{0\}, \quad \text { for all } i<m,
$$

there exists a homomorphism $h: B \rightarrow C$ such that $g=h \circ f$.

The following simple result summarizes some of the basic properties of flat and generic homomorphisms:

Lemma 4.2. Let $m$ be a natural number, let $A$ be a pseudo-simplicial space, let $A_{0}, \ldots, A_{m-1}, B, G$ be simple objects of $\mathcal{E}_{\mathrm{d}}$. Let $\alpha, \beta, \lambda$ be rational numbers such that $1 \leq \alpha \leq \beta$ and $1 \leq \lambda$.

(i) Every homomorphism $f: \bigoplus_{i<m} A_{i} \rightarrow B$ is $\lambda$-flat for some $\lambda \geq 1$. 
(ii) Every $\alpha$-flat homomorphism from a pseudo-simplicial space to a simple pseudo-simplicial space is $\beta$-flat.

(iii) Every $\beta$-generic homomorphism from a pseudo-simplicial space to a simple pseudo-simplicial space is $\alpha$-generic.

(iv) Suppose that $G$ is pseudo-simplicial, let $f: A \rightarrow G$ and $h: A \rightarrow B$ be homomorphisms, and let $\boldsymbol{g}: \operatorname{Id}_{\mathrm{c}} G \rightarrow \operatorname{Id}_{\mathrm{c}} B$ be a $\langle\vee, 0\rangle$-homomorphism such that $\operatorname{Id}_{\mathrm{c}} h=\boldsymbol{g} \circ \operatorname{Id}_{\mathrm{c}} f$. If $f$ is $\lambda$-generic and $h$ is $\lambda$-flat, then there exists a homomorphism $g: G \rightarrow B$ such that $\boldsymbol{g}=\operatorname{Id}_{\mathrm{c}} g$ and $h=g \circ f$.

The situation of (iv) above may be summarized by the following commutative diagrams:
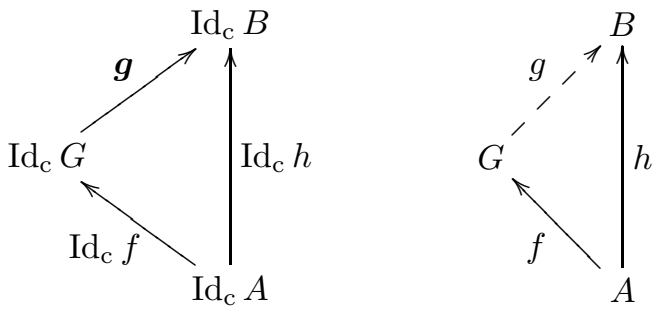

Proof. To prove (i) observe that if $f\left(1_{A_{i}}\right) \neq 0$, then it is an order-unit in $B$. Thus for any $i, j<m$ such that both $f\left(1_{A_{i}}\right)$ and $f\left(1_{A_{j}}\right)$ are nonzero, there exists $\lambda_{i, j} \in \mathbb{Q}^{++}$such that $f\left(1_{A_{i}}\right) \leq \lambda_{i, j} f\left(1_{A_{j}}\right)$. Now set $\lambda$ any positive rational number larger than 1 and all $\lambda_{i, j}$.

Claims (ii) and (iii) are straightforward.

To prove (iv) observe first that $\boldsymbol{g}$ has a lifting, that is, a homomorphism $g: G \rightarrow$ $B$ such that $\operatorname{Id}_{\mathrm{c}} g=\boldsymbol{g}$. Indeed, if $\boldsymbol{g}=0$, take $g=0$, while if $\boldsymbol{g}$ is the unique isomorphism from $\operatorname{Id}_{\mathrm{c}} G$ to $\operatorname{Id}_{\mathrm{c}} B$, any nonzero positive homomorphism from $G$ to $B$ is a lifting of $\boldsymbol{g}$, for example, if $G=\mathbb{Q}_{m}$ and if $b \in B^{++}$, the map $g: G \rightarrow B$ defined by the rule $g\left(x_{0}, \ldots, x_{m-1}\right)=\left(\sum_{i<m} x_{i}\right) b$, for all $x_{0}, \ldots, x_{m-1} \in \mathbb{Q}$.

Now, for (iv) above, if $h=0$, then any lifting $g$ of $\boldsymbol{g}$ satisfies the required conditions. So suppose that $h \neq 0$. Then, by the $\lambda$-genericity of $f$ and the $\lambda$ flatness of $h$, there exists a homomorphism $g: G \rightarrow B$ such that $h=g \circ f$. In particular, $g \neq 0$. Similarly, from $\operatorname{Id}_{\mathrm{c}} h=\boldsymbol{g} \circ \operatorname{Id}_{\mathrm{c}} f$ and $\operatorname{Id}_{\mathrm{c}} h \neq 0$ follows that $\boldsymbol{g} \neq 0$. Therefore, since both $G$ and $B$ are simple, $g$ lifts $\boldsymbol{g}$.

We shall now define the canonical generic maps. We are given a pseudo-simplicial space $A$ and a $\langle\vee, 0\rangle$-homomorphism $\boldsymbol{f}: \operatorname{Id}_{\mathrm{c}} A \rightarrow \mathbf{2}$. We shall construct a simple pseudo-simplicial space $G=\operatorname{Gen}(A, \boldsymbol{f})$ and a lifting $f: A \rightarrow G$ of $\boldsymbol{f}$ with respect to the $\operatorname{Id}_{\mathrm{c}}$ functor.

Put $A=\bigoplus_{i<m} A_{i}$, for a natural number $m$ and simple pseudo-simplicial spaces $A_{0}, \ldots, A_{m-1}$. We shall define a pseudo-simplicial space $G=\operatorname{Gen}(A, \boldsymbol{f})$ and, for every rational number $\lambda \geq 1$, a lifting $f_{\lambda}: A \rightarrow G$ of $f$ with respect to the $\operatorname{Id}_{c}$ functor.

For all $i<m$, let $T_{i}$ denote the canonical basis of $A_{i}$ (see Section 2). Hence the $T_{i}$, for $i<m$, are mutually disjoint finite sets and $\bigcup_{i<m} T_{i}$ is a vector space basis of $A$. Let $I=\left\{i<m \mid \boldsymbol{f}\left(A_{i}\right)=1\right\}$ (observe that $A_{i}$ is a compact ideal of $A$, thus it belongs to the domain of $\boldsymbol{f}$, see Lemma 2.3). Furthermore, we put

$$
T=\prod_{i \in I} T_{i}, \quad F=\mathcal{P}(I) \times T, \quad \text { and } \quad G=\mathbb{Q}_{F} .
$$


Observe that both $T$ and $F$ are finite, nonempty sets. For a rational number $\lambda \geq 1$, we define a linear map $f_{\lambda}: A \rightarrow \mathbb{Q}_{F}$ by its action on the elements of $\bigcup_{i<m} T_{i}$. For $i<m$ and $t \in T_{i}$, we define $f_{\lambda}(t)=0$ if $i \notin I$, while, if $i \in I$, we put

$$
f_{\lambda}(t)=\sum_{\substack{(X, \varphi) \in F \\ i \notin X, \varphi(i)=t}} 1_{(X, \varphi)}^{F}+\lambda \cdot \sum_{\substack{(X, \varphi) \in F \\ i \in X, \varphi(i)=t}} 1_{(X, \varphi)}^{F} .
$$

For all $i<m$, let $f_{i, \lambda}$ denote the restriction of $f_{\lambda}$ to $A_{i}$.

Lemma 4.3. The map $f_{i, \lambda}$ is a positive homomorphism from $A_{i}$ to $\mathbb{Q}_{F}$, for all $i<m$, and it is nonzero iff $i \in I$.

Proof. For $i \notin I, f_{i, \lambda}=0$. Suppose now that $i \in I$. It is trivial that $f_{i, \lambda}$ defines a positive homomorphism from $\left(A_{i}\right)_{\text {arch }} \cong \mathbb{Q}^{T_{i}}$ to $\mathbb{Q}^{F}$. To prove that it is also a nonzero positive homomorphism from $A_{i}$ to $\mathbb{Q}_{F}$, it is sufficient to prove that $f_{i, \lambda}\left(1_{A_{i}}\right)$ is an order-unit of $\mathbb{Q}^{F}$. By observing that $1_{A_{i}}=\sum_{t \in T_{i}} t$ and by using (4.1), we can compute:

$$
f_{i, \lambda}\left(1_{A_{i}}\right)=\sum_{\substack{(X, \varphi) \in F \\ i \notin X}} 1_{(X, \varphi)}^{F}+\lambda \cdot \sum_{\substack{(X, \varphi) \in F \\ i \in X}} 1_{(X, \varphi)}^{F} .
$$

Since all the components of this vector relatively to all $1_{(X, \varphi)}^{F}$, for $(X, \varphi) \in F$, are positive, $f_{i, \lambda}\left(1^{T_{i}}\right)$ is indeed an order-unit of $\mathbb{Q}^{F}$.

It follows from Lemma 4.3 that $f_{\lambda}$ is a positive homomorphism from $A$ to $\mathbb{Q}_{F}$. It follows immediately from Lemma 4.3 that $f_{\lambda}: A \rightarrow \mathbb{Q}_{F}$ is a lifting of the semilattice map $\boldsymbol{f}: \operatorname{Id}_{\mathrm{c}} A \rightarrow \mathbf{2}$. Indeed, if we take the canonical isomorphism from $\operatorname{Id}_{\mathrm{c}} \mathbb{Q}_{F}$ to $\mathbf{2}$, the following diagram

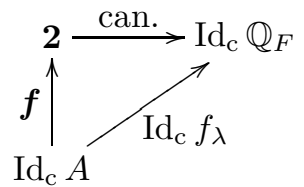

is commutative, since $f_{\lambda}\left[A_{i}\right] \neq\{0\}$ iff $\boldsymbol{f}\left(A_{i}\right)=1$, for all $i<m$.

Lemma 4.4. The homomorphism $f_{\lambda}$ is $\lambda$-flat.

Proof. For all $i \in I, f_{\lambda}\left(1_{A_{i}}\right)=f_{i, \lambda}\left(1_{A_{i}}\right)$ is a linear combination of the vectors $1_{(X, \varphi)}^{F}$, for $(X, \varphi) \in F$, with coefficients either 1 or $\lambda$ (see (4.1)). The conclusion follows.

We observe here the relevance of the definition of flatness by using $\smile^{\text {arch }}$ instead of $\asymp$ : indeed, the vectors $1_{(X, \varphi)}^{F}$, for $(X, \varphi) \in F$, do not belong to the positive cone of $\mathbb{Q}_{F}$ except if $F$ is a singleton.

Now we come to the main result of this section:

Lemma 4.5. The homomorphism $f_{\lambda}$ is $\lambda$-generic.

Proof. Let $C$ be a simple pseudo-simplicial space, let $g: \bigoplus_{i<m} A_{i} \rightarrow C$ be a $\lambda$-flat homomorphism such that $g\left[A_{i}\right] \neq\{0\}$ iff $f_{\lambda}\left[A_{i}\right] \neq\{0\}$ (that is, $i \in I$ ), for all $i<m$. Set again $A_{i}=\mathbb{Q}_{T_{i}}$ for every $i<m$. We define elements $a_{i}$ (for $i<m$ ) and $a_{(t)}$ (for $i<m$ and $t \in T_{i}$ ) of $C$ by

$$
a_{i}=g\left(1_{A_{i}}\right), \quad a_{(t)}=g(t) .
$$


Observe that $a_{i} \in C^{+}$while we can only say that $a_{(t)} \in C_{\mathrm{arch}}^{+}$. Moreover, $a_{i}=$ $\sum_{t \in T_{i}} a_{(t)}$. From the fact that $g$ is $\lambda$-flat follows that $a_{i} \asymp_{\lambda}^{\operatorname{arch}} a_{j}$ in $C$, for all $i$, $j \in I$, hence, by Lemma 3.2 applied to $C_{\text {arch }}$ (that has interpolation, because $C$ is pseudo-simplicial), there are elements $b_{X}$ (for $\left.X \in \mathcal{P}(I)\right)$ of $C_{\text {arch }}^{+}$such that

$$
a_{i}=\sum_{X \in \mathcal{P}_{i}^{*}(I)} b_{X}+\lambda \cdot \sum_{X \in \mathcal{P}_{i}(I)} b_{X}, \quad \text { for all } i \in I .
$$

Now, for fixed $i \in I$, we apply refinement to the equality

$$
\sum_{t \in T_{i}} a_{(t)}=\sum_{X \in \mathcal{P}_{i}^{*}(I)} b_{X}+\lambda \cdot \sum_{X \in \mathcal{P}_{i}(I)} b_{X},
$$

which holds in $C_{\text {arch }}^{+}$. We obtain elements $c_{X, i, t}\left(\right.$ for $(X, i) \in \mathcal{P}(I) \times I$ and $\left.t \in T_{i}\right)$ of $C_{\text {arch }}^{+}$such that

$$
\begin{aligned}
a_{(t)} & =\sum_{X \in \mathcal{P}_{i}^{*}(I)} c_{X, i, t}+\lambda \cdot \sum_{X \in \mathcal{P}_{i}(I)} c_{X, i, t} & & \text { (for all } \left.i \in I \text { and } t \in T_{i}\right) \\
b_{X} & =\sum_{t \in T_{i}} c_{X, i, t} & & \text { (for all } X \in \mathcal{P}(I)) .
\end{aligned}
$$

Now we apply Lemma 3.1 to the system of equations (4.4) in $C_{\mathrm{arch}}^{+}$. We find elements $d_{(X, \varphi)}($ for $(X, \varphi) \in F)$ of $C_{\text {arch }}^{+}$such that

$$
\begin{array}{rlrl}
b_{X} & =\sum_{\varphi \in T} d_{(X, \varphi)} & & (\text { for all } X \in \mathcal{P}(I)) \\
c_{X, i, t} & =\sum_{\substack{\varphi \in T \\
\varphi(i)=t}} d_{(X, \varphi)} & \left(\text { for all } X \in \mathcal{P}(I), i \in I, t \in T_{i}\right) .
\end{array}
$$

We define a linear map $h: \mathbb{Q}^{F} \rightarrow C$ by the rule

$$
h\left(1_{(X, \varphi)}^{F}\right)=d_{(X, \varphi)}, \quad \text { for all }(X, \varphi) \in F .
$$

Hence $h$ is a positive homomorphism from $\mathbb{Q}^{F}$ to $C_{\text {arch }}$. To verify that $h$ is a positive homomorphism from $\mathbb{Q}_{F}$ to $C$, it suffices to verify that $h\left(1^{F}\right) \in C^{+}$. This is trivial for $I=\varnothing\left(\right.$ then $\left.\mathbb{Q}_{F}=\mathbb{Q}\right)$. Suppose that $I \neq \varnothing$. We compute:

$$
\begin{aligned}
h\left(1^{F}\right)=\sum_{(X, \varphi) \in F} h\left(1_{(X, \varphi)}^{F}\right) & =\sum_{(X, \varphi) \in F} d_{(X, \varphi)} \\
& =\sum_{X \in \mathcal{P}(I)} b_{X} \quad(\text { by } 4.5) .
\end{aligned}
$$

Hence, it follows from (4.2) that $h\left(1^{F}\right) \asymp a_{i}$ in $C_{\text {arch }}$ for any $i \in I$. But $a_{i} \in C^{+}$, thus $h\left(1^{F}\right) \in C^{+}$.

It remains to prove that $h \circ f_{\lambda}(t)=g(t)$, for all $i<m$ and all $t \in T_{i}$. This is obvious if $i \notin I$, in which case both sides of the equality are zero. So suppose that 
$i$ belongs to $I$. We compute:

$$
\begin{aligned}
h \circ f_{\lambda}(t) & =\sum_{\substack{(X, \varphi) \in F \\
i \notin X, \varphi(i)=t}} d_{(X, \varphi)}+\lambda \cdot \sum_{\substack{(X, \varphi) \in F \\
i \in X, \varphi(i)=t}} d_{(X, \varphi)} & & \text { (by 4.1) and (4.7)) } \\
& =\sum_{X \in \mathcal{P}_{i}^{*}(I)} c_{X, i, t}+\lambda \cdot \sum_{X \in \mathcal{P}_{i}(I)} c_{X, i, t} & & \text { (by 4.6) }) \\
& =a_{(t)} & & \text { (by 44.3) }) \\
& =g(t) & & \text { (by the definition of } \left.a_{(t)}\right),
\end{aligned}
$$

which concludes the proof.

\section{Flat AND GENERIC homomorPhisms With ARBitraRY TARGET}

Definition 5.1. Let $n$ be a natural number, let $B_{0}, \ldots, B_{n-1}$ be simple pseudo-simplicial spaces, let $A$ be a pseudo-simplicial space, let $f: A \rightarrow \bigoplus_{j<n} B_{j}$ be a homomorphism. Write

$$
f(x)=\left(f_{0}(x), \ldots, f_{n-1}(x)\right), \quad \text { for all } x \in A,
$$

with $f_{j}: A \rightarrow B_{j}$, for all $j<n$. For any rational number $\lambda \geq 1$, we say that $f$ is

(i) $\lambda$-flat, if $f_{0}, \ldots, f_{n-1}$ are $\lambda$-flat.

(ii) $\lambda$-generic, if $f_{0}, \ldots, f_{n-1}$ are $\lambda$-generic.

Because of Lemma 2.3 the decomposition $B=\bigoplus_{j<n} B_{i}$ of $B$ into simple ideals is unique, hence the definition above is well-stated.

Part of Lemma 4.2 has a straightforward analogue for these more general definitions of flat and generic maps:

Lemma 5.2. Let $\alpha$ and $\beta$ be rational numbers such that $1 \leq \alpha \leq \beta$.

(i) Every homomorphism $f: A \rightarrow \bigoplus_{j<n} B_{j}$ is $\lambda$-flat for some $\lambda \geq 1$.

(ii) Every $\alpha$-flat homomorphism is $\beta$-flat.

(iii) Every $\beta$-generic homomorphism is $\alpha$-generic.

The definition of canonical generic homomorphisms of Section 4 extends easily to this new context, as follows.

Let $A$ be a pseudo-simplicial space, let $n$ be a natural number, let $\boldsymbol{f}: \operatorname{Id}_{\mathrm{c}} A \rightarrow \mathbf{2}^{n}$ be a $\langle\vee, 0\rangle$-homomorphism. So there are unique $\langle\vee, 0\rangle$-homomorphisms $\boldsymbol{f}_{j}: \operatorname{Id}_{\mathrm{c}} A \rightarrow$ $\mathbf{2}$, for $j<n$, such that

$$
\boldsymbol{f}(X)=\left(\boldsymbol{f}_{0}(X), \ldots, \boldsymbol{f}_{n-1}(X)\right), \quad \text { for all } X \in \operatorname{Id}_{\mathrm{c}} A .
$$

We put $G_{j}=\operatorname{Gen}\left(A, \boldsymbol{f}_{j}\right)$, for all $j<n$, and $G=\bigoplus_{j<n} G_{j}$. For any rational number $\lambda \geq 1$, we define the canonical $\lambda$-generic homomorphism $f_{\lambda}: A \rightarrow G$ by the rule

$$
f_{\lambda}(x)=\left(f_{0, \lambda}(x), \ldots, f_{n-1, \lambda}(x)\right), \quad \text { for all } x \in A,
$$

where $f_{j, \lambda}: A \rightarrow G_{j}$ is the canonical $\lambda$-generic map associated with $A$ and $\boldsymbol{f}_{j}$, for all $j<n$. It is trivial, by Lemmas 4.4 and 4.5 and by Definition [5.1] that $f_{\lambda}$ is both $\lambda$-flat and $\lambda$-generic.

Now define a map $\iota: \mathbf{2}^{n} \rightarrow \operatorname{Id}_{\mathrm{c}} G$ by

$$
\iota\left(i_{0}, i_{1}, \ldots, i_{n-1}\right)=\bigoplus_{j \in J} G_{j},
$$


where we put $J=\left\{j<n \mid i_{j}=1\right\}$. Since the diagram

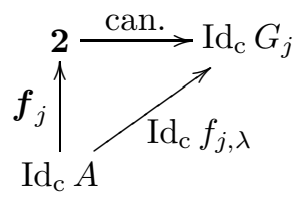

is commutative for any $j<n$, we get that also the diagram

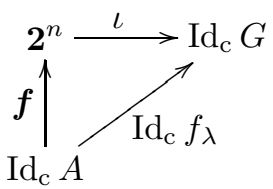

is commutative. Thus we have proved the following lemma.

Lemma 5.3. The identity $\iota \circ \boldsymbol{f}=\operatorname{Id}_{\mathrm{c}} f_{\lambda}$ holds. Thus the map $f_{\lambda}$ lifts $\boldsymbol{f}$.

The main result of this section is the following analogue of Lemma 4.2 (iv).

Lemma 5.4. Let $A, B, G$ be pseudo-simplicial spaces, let $f: A \rightarrow G$ and $h: A \rightarrow B$ be positive homomorphisms, and let $\boldsymbol{g}: \operatorname{Id}_{\mathrm{c}} G \rightarrow \operatorname{Id}_{\mathrm{c}} B$ be a $\langle\vee, 0\rangle$-homomorphism such that $\operatorname{Id}_{\mathrm{c}} h=\boldsymbol{g} \circ \operatorname{Id}_{\mathrm{c}} f$. Let $m, p$ be the natural numbers such that $\operatorname{Id}_{\mathrm{c}} A \cong \mathbf{2}^{m}$ and $\operatorname{Id}_{\mathrm{c}} G \cong \mathbf{2}^{p}$, and put $q=\min \left\{2^{m-1}, p\right\}$ if $m, p>0$, and $q=1$ otherwise. Let $\lambda \geq 1$ be a rational number. If $f$ is $q \lambda$-generic and $h$ is $\lambda$-flat, then there exists a homomorphism $g: G \rightarrow B$ such that $\boldsymbol{g}=\operatorname{Id}_{\mathrm{c}} g$ and $h=g \circ f$.

Proof. First of all, by decomposing $B$ as the direct sum of its simple ideals (see Lemma 2.3), it is easy to reduce the problem to the case where $B$ is simple. Furthermore, the problem is trivial if either $A$ or $G$ is trivial, so suppose from now on that both $m$ and $p$ are nonzero. Let $A=\bigoplus_{i<m} A_{i}$ and $G=\bigoplus_{j<p} G_{j}$ be the decompositions of $A$ and $G$ as the direct sums of their simple ideals. We write

$$
f(x)=\left(f_{0}(x), \ldots, f_{p-1}(x)\right), \quad \text { for all } x \in A,
$$

with homomorphisms $f_{j}: A \rightarrow G_{j}$, for $j<p$. Moreover, by using the natural identification of $\operatorname{Id}_{\mathrm{c}} G$ with $\prod_{j<p} \operatorname{Id}_{\mathrm{c}} G_{j}$, we can decompose $\boldsymbol{g}$ as

$$
\boldsymbol{g}\left(Y_{0}, \ldots, Y_{p-1}\right)=\bigvee_{j<p} \boldsymbol{g}_{j}\left(Y_{j}\right), \quad \text { for all }\left(Y_{0}, \ldots, Y_{p-1}\right) \in \prod_{j<p} \operatorname{Id}_{\mathrm{c}} G_{j},
$$

with $\langle\vee, 0\rangle$-homomorphisms $\boldsymbol{g}_{j}: \operatorname{Id}_{\mathrm{c}} G_{j} \rightarrow \operatorname{Id}_{\mathrm{c}} B$, for $j<p$. Hence $\operatorname{Id}_{\mathrm{c}} h=\bigvee_{j<p} \boldsymbol{h}_{j}$, where we put $\boldsymbol{h}_{j}=\boldsymbol{g}_{j} \circ \operatorname{Id}_{\mathrm{c}} f_{j}$, for all $j<p$. So $\boldsymbol{h}_{j}$ is a $\langle\vee, 0\rangle$-homomorphism from $\operatorname{Id}_{\mathrm{c}} A$ to $\operatorname{Id}_{\mathrm{c}} B$.

We define subsets $H, H_{j}$ (for $j<p$ ) of $m$ as follows:

$$
H=\left\{i<m \mid h\left[A_{i}\right] \neq\{0\}\right\}, \quad H_{j}=\left\{i<m \mid \boldsymbol{h}_{j}\left(A_{i}\right) \neq\{0\}\right\} .
$$

Put $\mathcal{H}=\left\{H_{j} \mid j<p\right\}$. Hence $H=\bigcup_{j<p} H_{j}=\bigcup \mathcal{H}$. Furthermore, put $n_{i}=$ $|\{K \in \mathcal{H} \mid i \in K\}|$, for all $i<m$. We make the following obvious observation:

$$
n_{i}=0 \text {, for all } i \notin H, \quad \text { and } \quad 1 \leq n_{i} \leq q, \quad \text { for all } i \in H .
$$

For any $K \in \mathcal{H}$, we define a homomorphism $h_{(K)}: A \rightarrow B$ by its restriction to each $A_{i}$, for $i<m$. First, we define the restriction of $h_{(K)}$ to $A_{i}$ as equal to zero if 
$i \notin K$. If $i \in K$, we put

$$
h_{(K)}(x)=\frac{1}{n_{i}} h(x), \quad \text { for all } x \in A_{i} .
$$

Hence, $h_{(K)}\left[A_{i}\right] \neq\{0\}$ iff $i \in K$, hence

$$
\operatorname{Id}_{\mathrm{c}} h_{\left(H_{j}\right)}=\boldsymbol{h}_{j}, \quad \text { for all } j<p .
$$

Furthermore, for all $i \in H$ and all $x \in A_{i}$,

$$
\sum_{K \in \mathcal{H}} h_{(K)}(x)=\sum_{\substack{K \in \mathcal{H} \\ i \in K}} \frac{1}{n_{i}} h(x)=h(x),
$$

whence we obtain the equality

$$
\sum_{K \in \mathcal{H}} h_{(K)}=h
$$

Next, we put $p_{j}=\left|\left\{j^{\prime}<p \mid H_{j}=H_{j^{\prime}}\right\}\right|$ and $h_{j}=\frac{1}{p_{j}} h_{\left(H_{j}\right)}$, for all $j<p$. Hence $p_{j}$ is a positive integer and, by (5.4), the following holds:

$$
\operatorname{Id}_{\mathrm{c}} h_{j}=\boldsymbol{h}_{j}, \quad \text { for all } j<p .
$$

Moreover, $\sum_{j<p} h_{j}=\sum_{K \in \mathcal{H}} h_{(K)}=h$ (the last equality follows from (5.5)). So we have obtained

$$
\sum_{j<p} h_{j}=h .
$$

Now we prove the following crucial claim:

Claim 1. The homomorphism $h_{j}$ is $q \lambda$-flat, for all $j<p$.

Proof of Claim. By the definition of $h_{j}$, it suffices to prove that $h_{(K)}$ is $q \lambda$-flat, for all $K \in \mathcal{H}$. For all $i \in K, h_{(K)}\left(1_{A_{i}}\right)=\frac{1}{n_{i}} h\left(1_{A_{i}}\right)$, so, for all $i, j \in K$,

$$
\begin{array}{rlr}
h_{(K)}\left(1_{A_{j}}\right)=\frac{1}{n_{j}} h\left(1_{A_{j}}\right) & \leq \text { arch } \frac{\lambda}{n_{j}} h\left(1_{A_{i}}\right) \quad \text { (because } h \text { is } \lambda \text {-flat) } \\
& =\frac{n_{i} \lambda}{n_{j}} h_{(K)}\left(1_{A_{i}}\right) & \\
& \leq n_{i} \lambda h_{(K)}\left(1_{A_{i}}\right) & \\
& \left.\leq q \lambda h_{(K)}\left(1_{A_{i}}\right) \quad \text { (by (5.2) }\right),
\end{array}
$$

which concludes the proof of the claim.

Claim 1.

Since $f$ is $q \lambda$-generic, it follows from Lemma 4.2 that for all $j<p$, there exists a homomorphism $g_{j}: G_{j} \rightarrow B$ such that $\operatorname{Id}_{\mathrm{c}} g_{j}=\boldsymbol{g}_{j}$ and $g_{j} \circ f_{j}=h_{j}$. We define $g: G \rightarrow B$ by the rule

$$
g\left(y_{0}, \ldots, y_{p-1}\right)=\sum_{j<p} g_{j}\left(y_{j}\right), \quad \text { for all }\left(y_{0}, \ldots, y_{p-1}\right) \in B .
$$

Then $\operatorname{Id}_{\mathrm{c}} g=\boldsymbol{g}$ and, by (5.7), $g \circ f=h$. 
Example 5.5. The following example shows that one cannot replace $q$ by 1 in the statement of Lemma 5.4. Let $h: \mathbb{Q}^{2} \rightarrow \mathbb{Q}$ be defined by the rule $h(x, y)=x+y$ (for all $x, y \in \mathbb{Q}$ ), and let $f: \mathbb{Q}^{2} \rightarrow A \oplus B$ be any 1-generic lifting of the map $\boldsymbol{f}: \mathbf{2}^{2} \rightarrow \mathbf{2}^{2}$ defined by the rule $\boldsymbol{f}(\boldsymbol{x}, \boldsymbol{y})=(\boldsymbol{x} \vee \boldsymbol{y}, \boldsymbol{y})$, with $A$ and $B$ being simple pseudo-simplicial spaces. Let $\boldsymbol{g}: \mathbf{2}^{2} \rightarrow \mathbf{2}$ be defined by the rule $\boldsymbol{g}(\boldsymbol{x}, \boldsymbol{y})=\boldsymbol{x} \vee \boldsymbol{y}$. By identifying $\operatorname{Id}_{\mathrm{c}}(A \oplus B)$ with $\mathbf{2}^{2}$ and $\operatorname{Id}_{\mathrm{c}} \mathbb{Q}$ with $\mathbf{2}$, the map $\boldsymbol{g}$ defines a $\langle\vee, 0\rangle$ homomorphism from $\operatorname{Id}_{\mathrm{c}}(A \oplus B)$ to $\operatorname{Id}_{\mathrm{c}} \mathbb{Q}$, and $\operatorname{Id}_{\mathrm{c}} h=\boldsymbol{g} \circ \operatorname{Id}_{\mathrm{c}} f$. Obviously, $h$ is 1-flat. However, we prove that there exists no lifting $g$ of $\boldsymbol{g}$ such that $h=g \circ f$. The situation is summarized on the diagrams below:
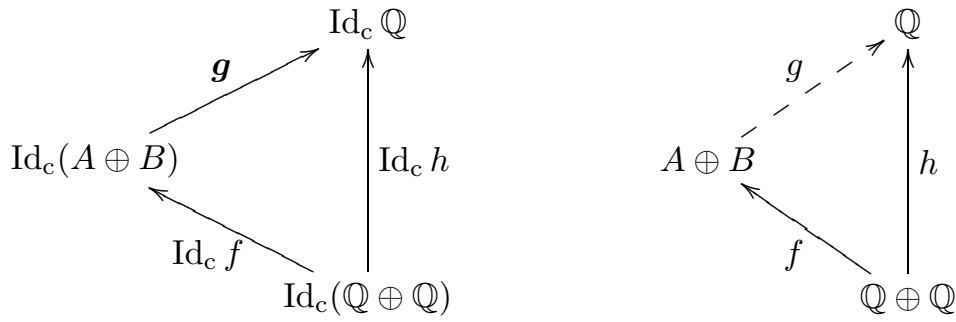

There are $a, c \in A^{++}$and $b \in B^{++}$such that

$$
f(x, y)=(x a+y c, y b), \quad \text { for all } x, y \in \mathbb{Q} .
$$

From the fact that $f$ is 1-flat follows that $a \asymp_{1}^{\text {arch }} c$ in $A$. Furthermore, there are positive, nonzero homomorphisms $u: A \rightarrow \mathbb{Q}$ and $v: B \rightarrow \mathbb{Q}$ such that

$$
g(x, y)=u(x)+v(y), \quad \text { for all } x \in A \text { and } y \in B .
$$

From $a \asymp_{1}^{\text {arch }} c$ follows that $u(a) \asymp_{1}^{\text {arch }} u(c)$, thus, since $u(a), u(c) \in \mathbb{Q}^{+}, u(a)=$ $u(c)$. However, by applying $h=g \circ f$ to $(1,0)$ and $(0,1)$ respectively, we obtain that $u(a)=1$ and $u(c)+v(b)=1$, hence $0<u(c)<u(a)=u(c)$, a contradiction.

We end this section with an easy lifting result:

Lemma 5.6. Let $E$ be a pseudo-simplicial space, let $m$ be a natural number, let $\boldsymbol{f}: \mathbf{2}^{m} \rightarrow \operatorname{Id}_{\mathrm{c}} E$ be a $\langle\vee, 0\rangle$-homomorphism. Then there exists a positive homomorphism $f: \mathbb{Q}^{m} \rightarrow E$ such that, if $\iota: \mathbf{2}^{m} \rightarrow \operatorname{Id}_{c} \mathbb{Q}^{m}$ denotes the canonical isomorphism, the equality $\boldsymbol{f}=\operatorname{Id}_{\mathrm{c}} f \circ \iota$ holds.

Proof. Let $E=\bigoplus_{j<n} E_{j}$ be the decomposition of $E$ as a direct sum of simple pseudo-simplicial spaces. Denote by $\left(\boldsymbol{e}_{i}\right)_{i<m}$ (resp., $\left.\left(e_{i}\right)_{i<m}\right)$ the canonical basis of $\mathbf{2}^{m}$ (resp., $\left.\mathbb{Q}^{m}\right)$. For all $i<m$, there exists a subset $J_{i}$ of $\{0,1, \ldots, n-1\}$ such that $\boldsymbol{f}\left(\boldsymbol{e}_{i}\right)=\bigoplus_{j \in J_{i}} E_{j}$. Let $f: \mathbb{Q}^{m} \rightarrow E$ be the linear map defined by $f\left(e_{i}\right)=\sum_{j \in J_{i}} 1_{E_{j}}$, for all $i<m$. Then, for all $i<m$,

$$
\left(\operatorname{Id}_{\mathrm{c}} f \circ \iota\right)\left(\boldsymbol{e}_{i}\right)=\left(\operatorname{Id}_{\mathrm{c}} f\right)\left(\mathbb{Q} e_{i}\right)=E\left(f\left(e_{i}\right)\right)=\bigoplus_{j \in J_{i}} E_{i}=\boldsymbol{f}\left(\boldsymbol{e}_{i}\right),
$$

whence $\operatorname{Id}_{\mathrm{c}} f \circ \iota=\boldsymbol{f}$.

\section{Pseudo-simplicial liftings of Dismantlable diagrams}

The existence of $\lambda$-generic mappings enables us to construct liftings of a large class of finite diagrams with respect to the functor $\operatorname{Id}_{c}$. The following definition is due to K. A. Baker, P. C. Fishburn, and F. S. Roberts [1]. 
Definition 6.1. A finite lattice $L$ of cardinality $n$ is called dismantlable if there is a chain $L_{1} \subset L_{2} \subset \cdots \subset L_{n}=L$ of sublattices of $L$ such that $\left|L_{i}\right|=i$ for every $i=1,2, \ldots, n$.

We shall use an extension of Definition 6.1 to partially ordered sets. We shall consider $\varnothing$ as a partially ordered set. For a finite partially ordered set $P$, an element $x$ of $P$ is doubly-irreducible, if $x$ has at most one upper cover and at most one lower cover. We denote by $\operatorname{Irr} P$ the set of all doubly-irreducible elements of $P$. For a subset $Q$ of $P$, let $Q \lessdot P$ be the statement that $Q=P \backslash\{x\}$ for some $x \in \operatorname{Irr} P$.

Definition 6.2. A finite partially ordered set $P$ of cardinality $n$ is dismantlable, if there exists a chain $\varnothing=P_{0} \lessdot P_{1} \lessdot \cdots \lessdot P_{n}=P$ of subsets of $P$.

Of course, a finite lattice is dismantlable iff it is dismantlable as a partially ordered set. On the other hand, every finite bounded dismantlable partially ordered set is a lattice as observed in D. Kelly and I. Rival [12]. Furthermore, a nonempty partially ordered set $P$ is dismantlable iff there exists $x \in \operatorname{Irr} P$ such that $P \backslash\{x\}$ is dismantlable.

There is a large supply of finite dismantlable lattices. For example, the following result was proved in [1]; another proof can be found in D. Kelly and I. Rival [12].

Theorem 6.3. Every finite planar lattice is dismantlable.

There are also non-planar modular dismantlable lattices, like the one diagrammed on Figure 1. On the other hand, every distributive dismantlable lattice is planar, see D. Kelly and I. Rival [1].

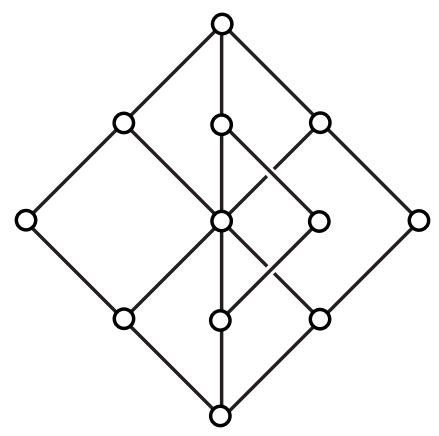

FIgURE 1. A dismantlable, modular, non-planar lattice

The main result of this section is the following.

Theorem 6.4. Every diagram $\Phi: P \rightarrow \mathcal{S}_{\mathrm{fb}}$ of finite Boolean semilattices indexed by a finite dismantlable partially ordered set $P$ has a pseudo-simplicial lifting $\Psi: P \rightarrow$ $\varepsilon_{\mathrm{ps}}$.

Proof. We shall proceed by induction on the cardinality of $P$. We may also assume without loss of generality that for every $x \in P$ we have $\Phi(x)=\mathbf{2}^{q}$ for some $q \in \mathbb{Z}^{+}$. If $P=\varnothing$ then the result is trivial.

Now assume that $P$ is nonempty. So there exists $x \in \operatorname{Irr} P$ such that $Q=P \backslash\{x\}$ is dismantlable. By the induction hypothesis, the restriction $\Phi^{\prime}$ of $\Phi$ to $Q$ has a pseudo-simplicial lifting $\Psi^{\prime}: Q \rightarrow \mathcal{E}_{\mathrm{ps}}$. So there are isomorphisms $\iota_{t}: \Phi(t) \rightarrow \operatorname{Id}_{\mathrm{c}}\left(\Psi^{\prime}(t)\right)$, 
for $t \in Q$, such that the following holds:

$$
\iota_{t} \circ \Phi\left(\varepsilon_{s, t}\right)=\operatorname{Id}_{\mathrm{c}}\left(\Psi^{\prime}\left(\varepsilon_{s, t}\right)\right) \circ \iota_{s}, \quad \text { for all } s \leq t \text { in } Q .
$$

Let $n \in \mathbb{Z}^{+}$such that $\Phi(x)=\mathbf{2}^{n}$.

Suppose first that $x$ is comparable to no element of $Q$. Then, in order to lift $\Phi$, it is sufficient to adjoin one lifting of $\Phi(x)$ to the diagram $\Psi^{\prime}$; take $\Psi(x)=\mathbb{Q}^{n}$.

So, from now on, suppose that $x$ can be compared to at least one element of $Q$. We denote by $u$ (resp., $v$ ) the unique lower cover (resp., upper cover) of $x$ in $P$ if it exists. By assumption, either $u$ or $v$ exists.

We start with the case where $x$ is a minimal element of $P$. Then $v$ exists, and, by Lemma [5.6 there exists a positive homomorphism $f: \mathbb{Q}^{n} \rightarrow \Psi^{\prime}(v)$ such that, if $\iota_{x}: \mathbf{2}^{n} \rightarrow \operatorname{Id}_{c} \mathbb{Q}^{n}$ denotes the canonical isomorphism, the equality

$$
\iota_{v} \circ \Phi\left(\varepsilon_{x, v}\right)=\operatorname{Id}_{\mathrm{c}} f \circ \iota_{x}
$$

holds. We define $\Psi(x)=\mathbb{Q}^{n}$, and, for every $t \in Q$ such that $x<t$, that is, $v \leq t$, we put $\Psi\left(\varepsilon_{x, t}\right)=\Psi^{\prime}\left(\varepsilon_{v, t}\right) \circ f$. It follows from (6.1) that the right half of the following diagram commutes,

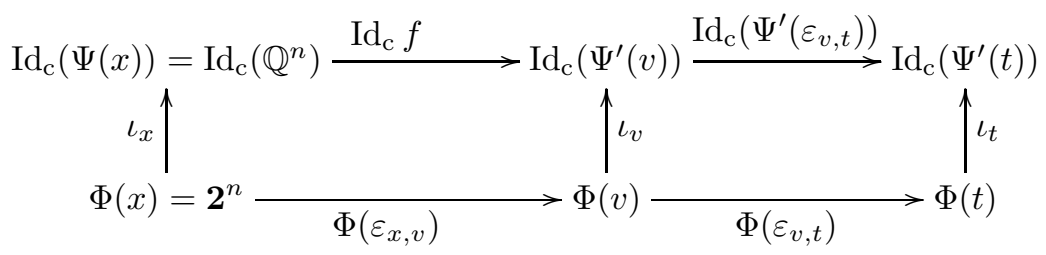

while the commutation of the left half of this diagram follows from (6.2). This settles the case where $x$ is a minimal element of $P$.

So, suppose, from now on, that $x$ is not minimal in $P$, so that $u$ exists. Let $m \in \mathbb{Z}^{+}$such that $\Phi(u)=\mathbf{2}^{m}$. Observe that $\boldsymbol{f}=\Phi\left(\varepsilon_{u, x}\right) \circ \iota_{u}^{-1}$ is a $\langle\vee, 0\rangle$-homomorphism from $\operatorname{Id}_{c}\left(\Psi^{\prime}(u)\right)$ to $\mathbf{2}^{n}$. Put $G=\operatorname{Gen}\left(\Psi^{\prime}(u), \boldsymbol{f}\right)$ (see Section 5), let $\iota_{x}: \mathbf{2}^{n} \rightarrow \operatorname{Id}_{c} G$ be the isomorphism given by (5.1). For any rational number $\mu \geq 1$, denote by $f_{\mu}: \Psi^{\prime}(u) \rightarrow G$ the canonical $\mu$-generic lifting of $\boldsymbol{f}$, see Section 5 Hence, by Lemma 5.3 the following diagram commutes,

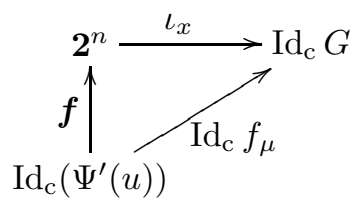

which means that the following equality holds:

$$
\operatorname{Id}_{c} f_{\mu} \circ \iota_{u}=\iota_{x} \circ \Phi\left(\varepsilon_{u, x}\right) \text {. }
$$

In case $x$ is maximal in $P$, set $\mu=1$. In case $x$ is not maximal in $P$, there exists, by Lemma [5.2(i) a rational number $\lambda \geq 1$ such that $\Psi^{\prime}\left(\varepsilon_{u, v}\right)$ is $\lambda$-flat. Put $q=\min \left\{2^{m-1}, n\right\}$ if $m, n>0, q=1$ otherwise, and put $\mu=q \lambda$. In both cases, we define $\Psi(x)=G$ and $\Psi\left(\varepsilon_{u, x}\right)=f_{\mu}$, the canonical $\mu$-generic lifting of $\boldsymbol{f}$. Hence for every $t \leq u$ we have to set $\Psi\left(\varepsilon_{t, x}\right)=f_{\mu} \circ \Psi^{\prime}\left(\varepsilon_{t, u}\right)$, and we need to check that 
the following diagram commutes:

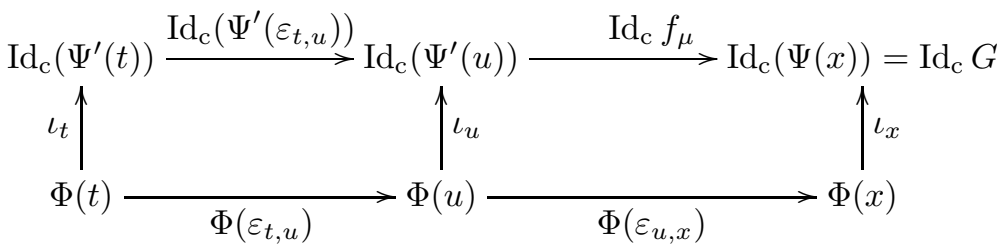

The commutativity of the left half of this diagram follows from (6.1), while the commutativity of the right half follows from (6.3). In particular, this completes the proof of the induction step if $x$ is the largest element of $P$.

If $x$ has a (unique) upper cover $v$ we continue as follows. We first observe that $\boldsymbol{g}=\iota_{v} \circ \Phi\left(\varepsilon_{x, v}\right) \circ \iota_{x}^{-1}$ is a $\langle\vee, 0\rangle$-homomorphism from $\operatorname{Id}_{\mathrm{c}} G$ to $\operatorname{Id}_{\mathrm{c}}\left(\Psi^{\prime}(v)\right)$. Since $\Psi^{\prime}\left(\varepsilon_{u, v}\right)$ is $\lambda$-flat and $\Psi^{\prime}\left(\varepsilon_{u, x}\right)=f_{\mu}$ is $q \lambda$-generic, Lemma 5.4 can be applied to the two following commutative diagrams:
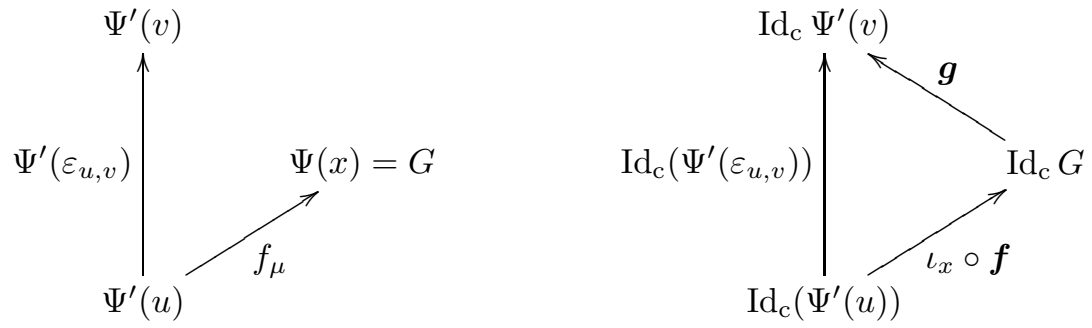

Thus there exists a positive homomorphism $g: G \rightarrow \Psi^{\prime}(v)$ such that $\operatorname{Id}_{\mathrm{c}} g=\boldsymbol{g}$ and $g \circ f_{\mu}=\Psi^{\prime}\left(\varepsilon_{u, v}\right)$. We define $\Psi\left(\varepsilon_{x, v}\right)=g$, thus for every $t \geq v$ we have to set $\Psi\left(\varepsilon_{x, t}\right)=\Psi^{\prime}\left(\varepsilon_{v, t}\right) \circ g$, and we need to check that the following diagram commutes:

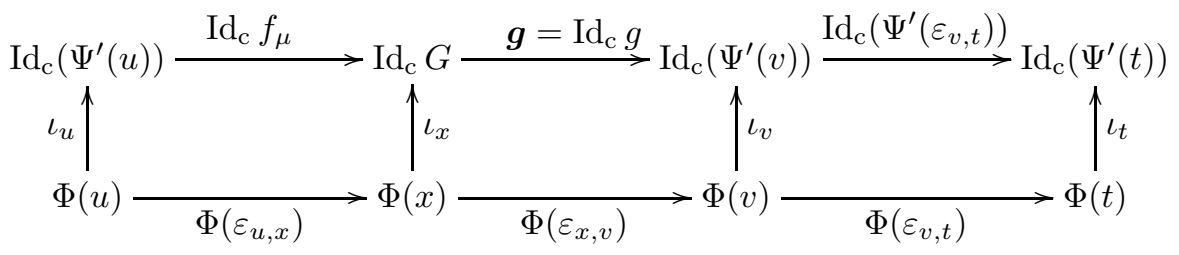

The commutativity of the right third of this diagram follows from (6.1), the commutativity of the left third follows from (6.3), and the commutativity of the middle third of this diagram follows from the fact that $\operatorname{Id}_{c} g=\boldsymbol{g}$ and the definition of $\boldsymbol{g}$. This completes the verification that the extension $\Psi$ of $\Psi^{\prime}$ thus defined is as required.

\section{Lifting $\langle\vee, 0\rangle$-Homomorphisms BetWeen Countable $\langle\vee, 0\rangle$-SEMilatTices}

The main result of this section is a wide generalization of Lemma [5.6

Theorem 7.1. Let $S$ be a countable distributive $\langle\vee, 0\rangle$-semilattice, let $H$ be a countable dimension vector space, let $\boldsymbol{f}: S \rightarrow \mathrm{Id}_{\mathrm{c}} H$ be a $\langle\vee, 0\rangle$-homomorphism. Then $f$ can be lifted, that is, there are a countable dimension vector space $G$, a positive homomorphism $f: G \rightarrow H$, and an isomorphism $\alpha: S \rightarrow \operatorname{Id}_{\mathrm{c}} G$ such that $\boldsymbol{f}=\left(\operatorname{Id}_{\mathrm{c}} f\right) \circ \alpha$. 
Proof. It follows from E. G. Effros, D. E. Handelman, and C.-L. Shen [3] (see also [6. Theorem 3.19]) that $H$ is the direct limit of a sequence of simplicial (partially ordered) groups, that is, groups of the form $\mathbb{Z}^{n}$, for $n \in \mathbb{Z}^{+}$. Since $H$ is a vector space, the isomorphism $H \cong H \otimes \mathbb{Q}$ implies that $H$ is, in fact, the direct limit of a sequence of simplicial vector spaces: more specifically, $H=\lim _{i \in \mathbb{Z}^{+}} H_{i}$, where $H_{i}=\mathbb{Q}^{n_{i}}$ for some $n_{i} \in \mathbb{Z}^{+}$, with transition maps $t_{i}: H_{i} \rightarrow H_{i+1}$ and limiting maps $t_{i}^{\prime}: H_{i} \rightarrow H$, both being positive homomorphisms, for all $i \in \mathbb{Z}^{+}$.

Furthermore, it follows from [8, Corollary 6.7(i)] that $S$ is the direct limit of a countable sequence of finite Boolean $\langle\vee, 0\rangle$-semilattices, say, $S=\varliminf_{\longrightarrow} \operatorname{li}_{\mathbb{Z}^{+}} \mathbf{2}^{m_{i}}$, with $m_{i} \in \mathbb{Z}^{+}$, transition maps $\boldsymbol{s}_{i}: \mathbf{2}^{m_{i}} \rightarrow \mathbf{2}^{m_{i+1}}$ and limiting maps $\boldsymbol{s}_{i}^{\prime}: \mathbf{2}^{m_{i}} \rightarrow S$, both being $\langle\vee, 0\rangle$-homomorphisms, for all $i \in \mathbb{Z}^{+}$. The situation may be visualized by the following commutative diagrams:
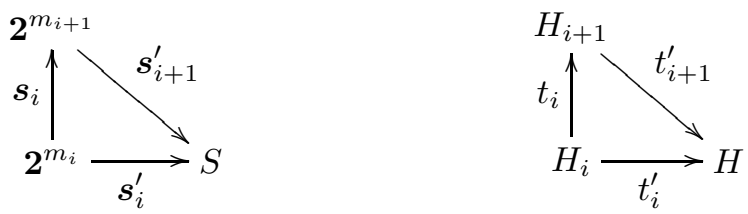

For all $i \in \mathbb{Z}^{+}$, the $\langle\vee, 0\rangle$-homomorphism $\boldsymbol{f} \circ \boldsymbol{s}_{i}^{\prime}: \mathbf{2}^{m_{i}} \rightarrow \operatorname{Id}_{\mathrm{c}} H \cong \lim _{j \in \mathbb{Z}^{+}} \operatorname{Id}_{\mathrm{c}} H_{j}$ factors through $\operatorname{Id}_{\mathrm{c}} H_{j}$ for some $j \in \mathbb{Z}^{+}$. Hence, by possibly replacing the sequence $\left(H_{j}\right)_{j \in \mathbb{Z}^{+}}$by some subsequence, we can suppose that $j=i$, allowing to factor $\boldsymbol{f}$ through a $\langle\vee, 0\rangle$-homomorphism $\boldsymbol{f}_{i}: \mathbf{2}^{m_{i}} \rightarrow \operatorname{Id}_{\mathrm{c}} H_{i}$. The situation may be visualized by the following commutative diagram, where the two parallel horizontal sequences are direct limits:

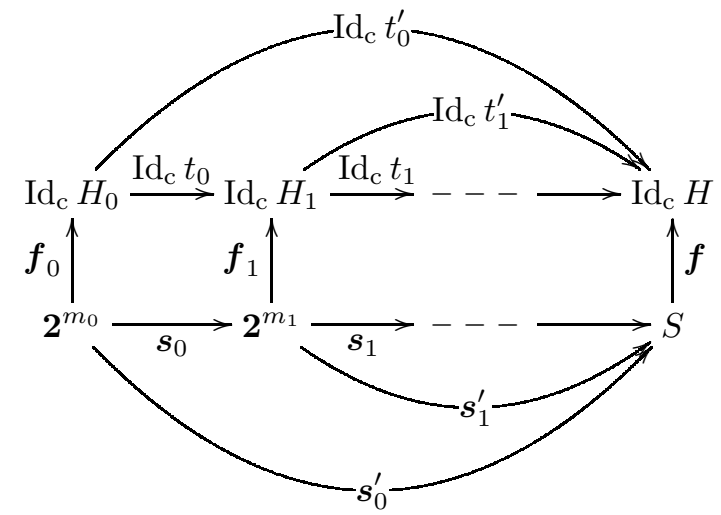

Now we construct, for $i \in \mathbb{Z}^{+}$, a certain pseudo-simplicial space $G_{i}$, together with an isomorphism $\alpha_{i}: \mathbf{2}^{m_{i}} \rightarrow \operatorname{Id}_{\mathrm{c}} G_{i}$ and a positive homomorphism $f_{i}: G_{i} \rightarrow H_{i}$ such that the following equality holds:

$$
\boldsymbol{f}_{i}=\left(\operatorname{Id}_{\mathrm{c}} f_{i}\right) \circ \alpha_{i} .
$$

For $i=0$, we pick $G_{0}, f_{0}$, and $\alpha_{0}$ satisfying (7.2); their existence is ensured by Lemma [5.6] Now suppose $G_{i}, f_{i}$, and $\alpha_{i}$ constructed satisfying (7.2) above, we construct $G_{i+1}, f_{i+1}$, and $\alpha_{i+1}$. First, by Lemma [5.2(i), there exists a rational number $\lambda \geq 1$ such that the homomorphism $t_{i} \circ f_{i}: G_{i} \rightarrow H_{i+1}$ is $\lambda$-flat. We put $q=\min \left\{2^{m_{i}-1}, n_{i+1}\right\}$ if $m_{i}, n_{i+1}>0$ and $q=1$ otherwise. Furthermore, we put $G_{i+1}=\operatorname{Gen}\left(G_{i}, s_{i} \circ \alpha_{i}^{-1}\right)$, and we let $s_{i}: G_{i} \rightarrow G_{i+1}$ be the canonical 
$q \lambda$-generic lifting of $\boldsymbol{s}_{i} \circ \alpha_{i}^{-1}$. In particular, Lemma 5.3 yields us an isomorphism $\alpha_{i+1}: \mathbf{2}^{m_{i+1}} \rightarrow \operatorname{Id}_{\mathrm{c}} G_{i+1}$ such that the following equality holds:

$$
\operatorname{Id}_{\mathrm{c}} s_{i}=\alpha_{i+1} \circ s_{i} \circ \alpha_{i}^{-1} \text {. }
$$

It follows from Lemma 5.4 that there exists a positive homomorphism $f_{i+1}: G_{i+1} \rightarrow$ $H_{i+1}$ such that $\operatorname{Id}_{\mathrm{c}} f_{i+1}=\boldsymbol{f}_{i+1} \circ \alpha_{i+1}^{-1}$ and $f_{i+1} \circ s_{i}=t_{i} \circ f_{i}$. The situation may be visualized by the following commutative diagrams:
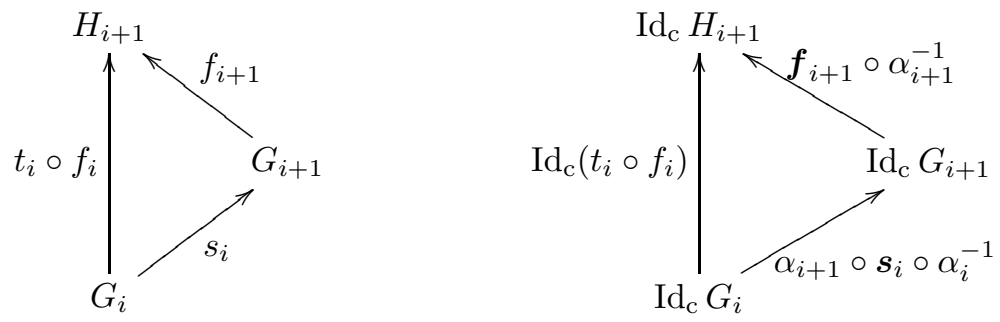

Hence the following diagrams are commutative:
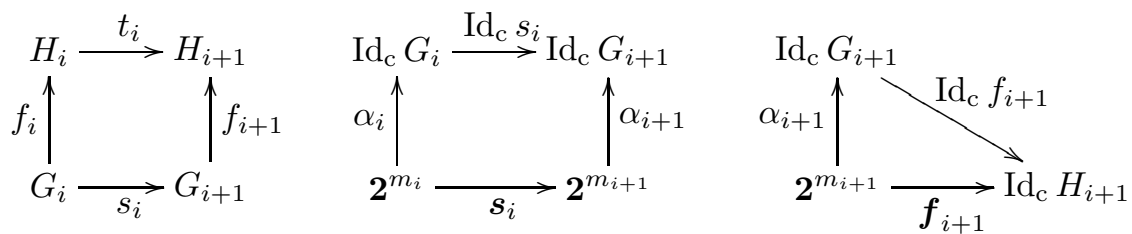

We let $G$ be the direct limit of the sequence $\left(G_{i}\right)_{i \in \mathbb{Z}^{+}}$, with transition maps $s_{i}: G_{i} \rightarrow$ $G_{i+1}$ and limiting maps $s_{i}^{\prime}: G_{i} \rightarrow G$, for all $i \in \mathbb{Z}^{+}$. Hence $G$ is a dimension vector space. Furthermore, since the functor $\operatorname{Id}_{\mathrm{c}}$ preserves direct limits, the commutativity of the left diagram and the middle diagram in (7.4) imply the existence of a positive homomorphism $f: G \rightarrow H$ and an isomorphism $\alpha: S \rightarrow \operatorname{Id}_{c} G$ such that the following infinite diagrams are commutative:

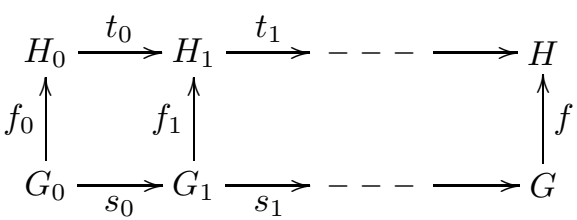

and

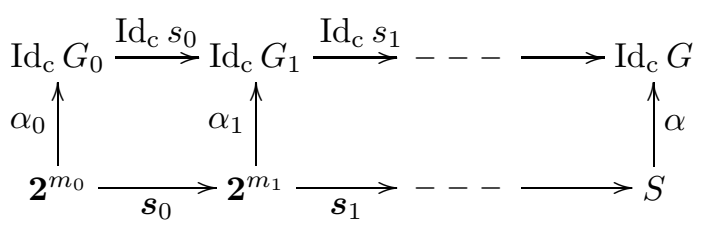

(For sake of clarity, we do not represent on these diagrams the transition maps corresponding to the direct limits in the rows of (17.5), (77.6).) To verify the equality $\left(\operatorname{Id}_{\mathrm{c}} f\right) \circ \alpha=\boldsymbol{f}$, it suffices to verify that $\left(\operatorname{Id}_{\mathrm{c}} f_{i}\right) \circ \alpha_{i}=\boldsymbol{f}_{i}$ holds, for all $i \in \mathbb{Z}^{+}$, which is exactly (7.2).

In particular, it follows from Theorem 7.1 that every countable distributive $\langle\vee, 0\rangle$-semilattice is isomorphic to $\operatorname{Id}_{c} G$ for some dimension vector space $G$, but 
this is of course much easier to prove directly, see [8, Theorem 5.2]. As an immediate consequence of this and Theorem 7.1 we record the following:

Corollary 7.2. Let $S$ and $T$ be countable distributive $\langle\vee, 0\rangle$-semilattices, let $\boldsymbol{f}: S \rightarrow$ $T$ be a $\langle\vee, 0\rangle$-homomorphism. Then $\boldsymbol{f}$ can be lifted, that is, there are countable dimension vector spaces $G$ and $H$ together with a positive homomorphism $f: G \rightarrow H$ and isomorphisms $\alpha: S \rightarrow \operatorname{Id}_{\mathrm{c}} G$ and $\beta: T \rightarrow \operatorname{Id}_{\mathrm{c}} H$ such that $\left(\operatorname{Id}_{\mathrm{c}} f\right) \circ \alpha=\beta \circ f$.

Corollary 7.2 cannot be extended to uncountable semilattices, see Example 11.1

In order to obtain a ring-theoretical version of Corollary [7.2 we shall need the following more precise well-known form of the isomorphism (0.1), which is essentially contained in [5, Theorem 15.20]:

Lemma 7.3. Let $K$ be a field. For a unital locally matricial $K$-algebra $R$, we let $\eta_{R}: \operatorname{Id}_{\mathrm{c}}\left(K_{0}(R)\right) \rightarrow \operatorname{Id}_{\mathrm{c}} R$ be the map defined by the rule

$$
\eta_{R}(I)=\{x \in R \mid[x R] \in I\}, \quad \text { for all } I \in \operatorname{Id}_{\mathrm{c}}\left(K_{0}(R)\right)
$$

(we let $[x R]$ denote the isomorphism type of $x R$ ). Then $\eta_{R}$ is a semilattice isomorphism and the rule $R \mapsto \eta_{R}$ defines a natural equivalence from the functor $\operatorname{Id}_{\mathrm{c}} \circ K_{0}$ $\left(\mathrm{Id}_{\mathrm{c}}\right.$ is meant on dimension groups) to the functor $\mathrm{Id}_{\mathrm{c}}$ (defined on locally matricial $K$-algebras). This means that for any unital locally matricial $K$-algebras $A$ and $B$ and any unital $K$-algebra homomorphism $f: A \rightarrow B$, the following diagram is commutative:

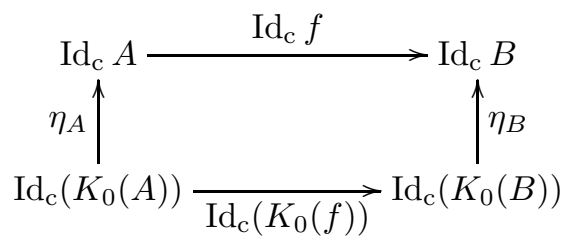

We can now state the ring-theoretical analogue of Corollary 7.2

Corollary 7.4. Let $S$ and $T$ be countable distributive $\langle\vee, 0\rangle$-semilattices (resp., countable distributive $\langle\vee, 0\rangle$-semilattices with unit), let $f: S \rightarrow T$ be a $\langle\vee, 0\rangle$-homomorphism (resp., a $\langle\vee, 0,1\rangle$-homomorphism), let $K$ be a field. Then there are countably dimensional locally matricial (resp., countably dimensional locally matricial unital) $K$-algebras $A$ and $B$, a K-algebra homomorphism (resp., a unital K-algebra homomorphism) $f: A \rightarrow B$, and isomorphisms $\alpha: S \rightarrow \operatorname{Id}_{\mathrm{c}} A$ and $\beta: T \rightarrow \operatorname{Id}_{\mathrm{c}} B$ such that $\left(\operatorname{Id}_{\mathrm{c}} f\right) \circ \alpha=\beta \circ \boldsymbol{f}$.

Proof. We first deal with the case of unital homomorphisms, so both $S$ and $T$ have unit and $\boldsymbol{f}(1)=1$. By Corollary 7.2 there are countable dimension vector spaces $G$ and $H$ together with a positive homomorphism $f^{\prime}: G \rightarrow H$ and isomorphisms $\alpha^{\prime}: S \rightarrow \operatorname{Id}_{\mathrm{c}} G$ and $\beta^{\prime}: T \rightarrow \operatorname{Id}_{\mathrm{c}} H$ such that $\left(\operatorname{Id}_{\mathrm{c}} f^{\prime}\right) \circ \alpha^{\prime}=\beta^{\prime} \circ \boldsymbol{f}$. Since both $S$ and $T$ have a largest element, both $G$ and $H$ have an order-unit, that we will denote respectively by $1_{G}$ and $1_{H}$. Moreover, from $\boldsymbol{f}(1)=1$ follows that we may take $f^{\prime}\left(1_{G}\right)=1_{H}$. By the Elliott, Effros, Handelman, and Shen Theorem (see [4, 3, 5]), there are countably dimensional, locally matricial, unital $K$-algebras $A, B$ and (normalized) isomorphisms $\alpha^{\prime \prime}:\left(G, 1_{G}\right) \rightarrow\left(K_{0}(A),[A]\right)$ and $\beta^{\prime \prime}:\left(H, 1_{H}\right) \rightarrow$ $\left(K_{0}(B),[B]\right)$. By [7 Lemma 1.3], there exists a unital $K$-algebra homomorphism 
$f: A \rightarrow B$ such that $K_{0}(f)=\beta^{\prime \prime} \circ f^{\prime} \circ \alpha^{\prime \prime-1}$. Hence the following diagram commutes:

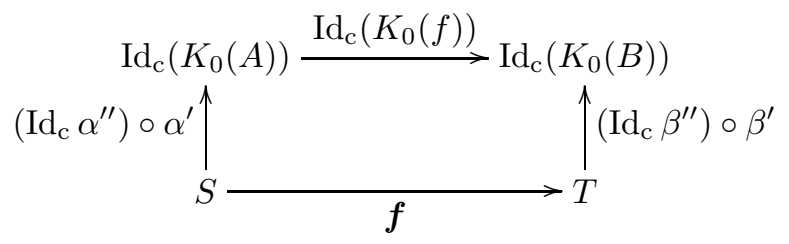

By Lemma [7.3 the following diagram commutes,

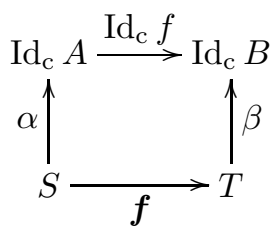

where we put $\alpha=\eta_{A} \circ\left(\operatorname{Id}_{\mathrm{c}} \alpha^{\prime \prime}\right) \circ \alpha^{\prime}$ and $\beta=\eta_{B} \circ\left(\operatorname{Id}_{\mathrm{c}} \beta^{\prime \prime}\right) \circ \beta^{\prime}$. This solves the case with unit.

In the case without unit, we put $S^{\prime}=S \cup\{1\}, T^{\prime}=T \cup\{1\}$ (where 1 is the largest element), and we extend $\boldsymbol{f}$ to a map $\boldsymbol{f}^{\prime}: S^{\prime} \rightarrow T^{\prime}$ by putting $\boldsymbol{f}^{\prime}(1)=1$. We let $f^{\prime}: A^{\prime} \rightarrow B^{\prime}$, together with isomorphisms $\alpha^{\prime}: S^{\prime} \rightarrow \operatorname{Id}_{\mathrm{c}} A^{\prime}$ and $\beta^{\prime}: T^{\prime} \rightarrow \operatorname{Id}_{\mathrm{c}} B^{\prime}$, lift $\boldsymbol{f}^{\prime}$, by using the result of the case with unit. Then we put

$$
\begin{aligned}
& A=\left\{x \in A^{\prime} \mid \alpha^{\prime-1}\left(A^{\prime} x A^{\prime}\right) \in S\right\}, \\
& B=\left\{y \in B^{\prime} \mid \beta^{\prime-1}\left(B^{\prime} y B^{\prime}\right) \in T\right\},
\end{aligned}
$$

so $A$ (resp., $B$ ) is an ideal of $A^{\prime}$ (resp., $B^{\prime}$ ). Then we let $f$ be the restriction of $f^{\prime}$ from $A$ to $B$ and $\alpha: S \rightarrow \operatorname{Id}_{\mathrm{c}} A$ (resp., $\beta: T \rightarrow \operatorname{Id}_{\mathrm{c}} B$ ) be the restrictions of $\alpha^{\prime}$ and $\beta^{\prime}$ to $S$ and $T$, respectively.

Every locally matricial algebra $R$ is a von Neumann regular ring, and then $\operatorname{Id}_{\mathrm{c}} R$ is isomorphic to $\operatorname{Con}_{\mathrm{c}} \mathcal{L}(R)$, the semilattice of compact congruences of the sectionally complemented, modular lattice $\mathcal{L}(R)$ of all principal right ideals of $R$, see [18, Corollary 4.4]. (A lattice $L$ with zero is sectionally complemented, if for all $a \leq b$ in $L$, there exists $x \in L$ such that $a \wedge x=0$ and $a \vee x=b$.) The proof of [18, Corollary 4.4] does not involve the unit, and, for a finite field $K$, every locally matricial $K$-algebra $R$ is locally finite, thus so is the lattice $\mathcal{L}(R)$. Thus similar methods as those used in the proof of Corollary 7.4 yield easily the following lattice-theoretical analogue of Corollary 7.4 .

Corollary 7.5. Let $S$ and $T$ be countable distributive $\langle\vee, 0\rangle$-semilattices, let $\boldsymbol{f}: S \rightarrow$ $T$ be a $\langle\vee, 0\rangle$-homomorphism. Then there are locally finite, sectionally complemented, modular lattices $L$ and $M$ together with a $\langle\vee, \wedge, 0\rangle$-homomorphism $f: L \rightarrow$ $M$ and isomorphisms $\alpha: S \rightarrow \operatorname{Con}_{\mathrm{c}} L$ and $\beta: T \rightarrow \operatorname{Con}_{\mathrm{c}} M$ such that $\left(\operatorname{Con}_{\mathrm{c}} f\right) \circ \alpha=$ $\beta \circ f$. Furthermore, if both $S$ and $T$ have a unit, then one may take both $L$ and $M$ with unit and $f(1)=1$.

The lattices $L$ and $M$ are more than just locally finite and sectionally complemented, for example, every finite subset of $L$ is contained in a finite, sectionally complemented, modular sublattice of $L$. 


\section{1-DimENSIONAL LIFTING FAILS FOR THE Id $_{\mathrm{c}}$ FUNCTOR AND $\langle\vee, 0\rangle$-SEMILATTICE EMBEDDINGS}

The following example is a modification of an earlier example of the second author, and it has been communicated to the authors by P. Růžička.

Example 8.1. There exists a dimension group $G$ together with a $\langle\vee, 0\rangle$-embedding $\mu: \operatorname{Id}_{\mathrm{c}} G \hookrightarrow \mathbf{2}^{2}$ that cannot be lifted. More precisely, there are no partially ordered abelian group $H$, no positive homomorphism $f: G \rightarrow H$, and no isomorphism $\alpha: \operatorname{Id}_{\mathrm{c}} H \rightarrow \mathbf{2}^{2}$ such that $\mu=\alpha \circ \operatorname{Id}_{\mathrm{c}} f$.

Proof. Let $G=\mathbb{Z} \times_{\text {lex }} \mathbb{Z}$ be the lexicographical product of the chain $\mathbb{Z}$ of integers with itself, put $a=(0,1)$ and $b=(1,0)$. Hence $n a \leq b$, for all $n \in \mathbb{Z}^{+}$, which we write $a \ll b$. So $\operatorname{Id}_{\mathrm{c}} G \cong \mathbf{3}$, the three-element chain. Denote further by $x, y$ the atoms of $2^{2}$.

Let $\mu: \operatorname{Id}_{\mathrm{c}} G \rightarrow \mathbf{2}^{2}$ be defined by the rule $\mu(G(0))=0, \mu(G(a))=x$, and $\mu(G(b))=1$. Observe that $\mu$ is a $\langle\vee, 0\rangle$-embedding. Suppose that there are $H, f$, and $\alpha$ as in the statement above.

Put $a^{\prime}=f(a)$ and $b^{\prime}=f(b)$. From $a \ll b$ follows that $a^{\prime} \ll b^{\prime}$. From $\alpha\left(H\left(b^{\prime}\right)\right)=$ $1=x \vee y$ follows that there are $c, d \in H^{+}$such that $\alpha(H(c))=x, \alpha(H(d))=y$, and $b^{\prime} \leq c+d$. Since $\alpha\left(H\left(a^{\prime}\right)\right)=x=\alpha(H(c))$ and $\alpha$ is an isomorphism, the relation $a^{\prime} \asymp c$ holds, thus there exists $n \in \mathbb{N}$ such that $c \leq n a^{\prime}$. Put $u=b^{\prime}-n a^{\prime}$. Since $0<a^{\prime} \ll b^{\prime}$, we get $0<a^{\prime} \ll u$ and $H(u)=H\left(b^{\prime}\right) ;$ thus $\alpha(H(u))=1$. But from $c \leq n a^{\prime}$ we get $u=b^{\prime}-n a^{\prime} \leq b^{\prime}-c \leq d$. Thus $H(u) \subseteq H(d)$ and $1=\alpha(H(u)) \leq \alpha(H(d))=y$, a contradiction.

Remark 8.2. No mention of the interpolation property has been made here, neither for $G$ nor for $H$.

\section{A Finite Diagram Without ANY Lifting BY INTERPolation GRoups}

The "diagram" of the following example is indexed not by a partially ordered set, but by a category, namely the category with exactly one object and one nontrivial idempotent endomorphism.

Example 9.1. Let $\boldsymbol{t}: \mathbf{2}^{2} \rightarrow \mathbf{2}^{2}$ defined by the rule $\boldsymbol{t}(\boldsymbol{x}, \boldsymbol{y})=(\boldsymbol{x} \vee \boldsymbol{y}, \boldsymbol{y})$ (for all $\boldsymbol{x}, \boldsymbol{y}<2)$. Then there is no lifting $t$ of $\boldsymbol{t}$, between interpolation groups, such that $t^{2}=t$.

Proof. Suppose otherwise. Let $G$ be an interpolation group such that $t$ is an endomorphism of $G$. Since $G$ is an interpolation group and $G$ lifts $2^{2}, G$ can be decomposed as $E \times F$, where $E$ and $F$ are simple interpolation groups, and $t$ is given by

$$
t(x, y)=(\alpha x+\beta y, \gamma y), \quad \text { for all }(x, y) \in E \times F,
$$

where $\alpha: E \rightarrow E, \beta: F \rightarrow E$, and $\gamma: F \rightarrow F$ are nonzero positive homomorphisms. The equality $t=t^{2}$ can then be expressed in matrix form as

$$
\left(\begin{array}{ll}
\alpha & \beta \\
0 & \gamma
\end{array}\right)=\left(\begin{array}{ll}
\alpha & \beta \\
0 & \gamma
\end{array}\right)\left(\begin{array}{cc}
\alpha & \beta \\
0 & \gamma
\end{array}\right)=\left(\begin{array}{cc}
\alpha^{2} & \alpha \beta+\beta \gamma \\
0 & \gamma^{2}
\end{array}\right)
$$

that is,

$$
\alpha^{2}=\alpha, \quad \gamma^{2}=\gamma, \quad \text { and } \quad \alpha \beta+\beta \gamma=\beta
$$


Hence, by repeated uses of (9.1), we obtain the equalities

$$
\begin{aligned}
\beta & =\alpha \beta+\beta \gamma \\
& =\alpha(\alpha \beta+\beta \gamma)+(\alpha \beta+\beta \gamma) \gamma \\
& =\alpha^{2} \beta+2 \alpha \beta \gamma+\beta \gamma^{2} \\
& =\beta+2 \alpha \beta \gamma,
\end{aligned}
$$

so we obtain that

$$
2 \alpha \beta \gamma=0 \text {. }
$$

However, $E$ and $F$ are simple partially ordered abelian groups and $\alpha, \beta, \gamma$ are nonzero positive homomorphisms, in particular, $(2 \alpha \beta \gamma)\left[F^{++}\right] \subseteq E^{++}$, which contradicts (9.2).

10. A diagram of Finite Boolean $\langle\vee, 0\rangle$-SEMilattices AND $\langle\vee, 0\rangle$-EMBEDDINGS WITHOUT ANY SIMPLICIAL LIFTING

Example 10.1. Let $\boldsymbol{f}: \mathbf{2}^{2} \hookrightarrow \mathbf{2}^{3}$ and $\boldsymbol{g}, \boldsymbol{h}: \mathbf{2}^{3} \hookrightarrow \mathbf{2}^{4}$ be the maps defined by

$$
\begin{aligned}
\boldsymbol{f}(\boldsymbol{x}, \boldsymbol{y}) & =(\boldsymbol{x}, \boldsymbol{y}, \boldsymbol{x} \vee \boldsymbol{y}), \\
\boldsymbol{g}(\boldsymbol{u}, \boldsymbol{v}, \boldsymbol{w}) & =(\boldsymbol{u}, \boldsymbol{v}, \boldsymbol{w}, \boldsymbol{u} \vee \boldsymbol{w}), \\
\boldsymbol{h}(\boldsymbol{u}, \boldsymbol{v}, \boldsymbol{w}) & =(\boldsymbol{u}, \boldsymbol{v}, \boldsymbol{w}, \boldsymbol{v} \vee \boldsymbol{w}),
\end{aligned}
$$

for all $\boldsymbol{x}, \boldsymbol{y}, \boldsymbol{u}, \boldsymbol{v}, \boldsymbol{w} \in\{0,1\}$. Observe that $\boldsymbol{g} \circ \boldsymbol{f}=\boldsymbol{h} \circ \boldsymbol{f}$, see the diagram (10.1).

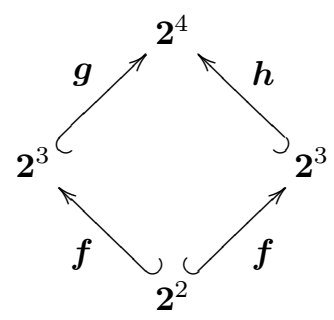

Then the diagram (10.1) has no lifting that uses only simplicial partially ordered vector spaces, that is, a lifting of the form

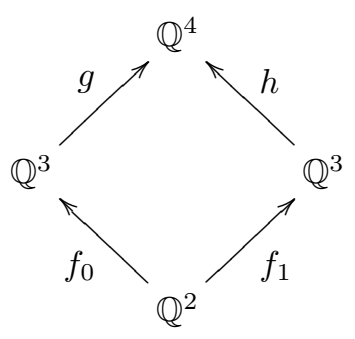


Proof. Suppose otherwise. We identify the maps $f_{0}, f_{1}, g$, and $h$ of the diagram (10.2) with their matrices, that have the following form:

$$
\begin{aligned}
f_{0}=\left(\begin{array}{cc}
\alpha & 0 \\
0 & \beta \\
\xi & \eta
\end{array}\right), & f_{1}=\left(\begin{array}{cc}
\alpha^{\prime} & 0 \\
0 & \beta^{\prime} \\
\xi^{\prime} & \eta^{\prime}
\end{array}\right), \\
g=\left(\begin{array}{ccc}
a & 0 & 0 \\
0 & b & 0 \\
0 & 0 & c \\
u & 0 & w
\end{array}\right), & h=\left(\begin{array}{ccc}
a^{\prime} & 0 & 0 \\
0 & b^{\prime} & 0 \\
0 & 0 & c^{\prime} \\
0 & v^{\prime} & w^{\prime}
\end{array}\right),
\end{aligned}
$$

with positive rational numbers $\alpha, \beta, \xi, \eta, \alpha^{\prime}, \beta^{\prime}, \xi^{\prime}, \eta^{\prime}, a, b, c, u, w, a^{\prime}, b^{\prime}, c^{\prime}, v^{\prime}$, $w^{\prime}$. Hence the equality $g \circ f_{0}=h \circ f_{1}$ takes the form

$$
\left(\begin{array}{ccc}
a & 0 & 0 \\
0 & b & 0 \\
0 & 0 & c \\
u & 0 & w
\end{array}\right)\left(\begin{array}{cc}
\alpha & 0 \\
0 & \beta \\
\xi & \eta
\end{array}\right)=\left(\begin{array}{ccc}
a^{\prime} & 0 & 0 \\
0 & b^{\prime} & 0 \\
0 & 0 & c^{\prime} \\
0 & v^{\prime} & w^{\prime}
\end{array}\right)\left(\begin{array}{cc}
\alpha^{\prime} & 0 \\
0 & \beta^{\prime} \\
\xi^{\prime} & \eta^{\prime}
\end{array}\right),
$$

that is,

$$
\left(\begin{array}{cc}
a \alpha & 0 \\
0 & b \beta \\
c \xi & c \eta \\
u \alpha+w \xi & w \eta
\end{array}\right)=\left(\begin{array}{cc}
a^{\prime} \alpha^{\prime} & 0 \\
0 & b^{\prime} \beta^{\prime} \\
c^{\prime} \xi^{\prime} & c^{\prime} \eta^{\prime} \\
w^{\prime} \xi^{\prime} & v^{\prime} \beta^{\prime}+w^{\prime} \eta^{\prime}
\end{array}\right)
$$

Hence, $\frac{\eta^{\prime}}{\xi^{\prime}}=\frac{c^{\prime} \eta^{\prime}}{c^{\prime} \xi^{\prime}}=\frac{c \eta}{c \xi}=\frac{\eta}{\xi}$, while $\frac{\eta^{\prime}}{\xi^{\prime}}=\frac{w^{\prime} \eta^{\prime}}{w^{\prime} \xi^{\prime}}<\frac{v^{\prime} \beta^{\prime}+w^{\prime} \eta^{\prime}}{w^{\prime} \xi^{\prime}}=\frac{w \eta}{u \alpha+w \xi}<\frac{w \eta}{w \xi}=$ $\frac{\eta}{\xi}$, a contradiction.

Remark 10.2. We have used only the last two rows of the matrices in (10.3), the rest is there to ensure that $\boldsymbol{f}, \boldsymbol{g}$, and $\boldsymbol{h}$ are embeddings.

\section{A semilattice map Without Lifting}

The following counterexample shows that Corollary 7.2 that states that every $\langle\vee, 0\rangle$-homomorphism between countable distributive $\langle\vee, 0\rangle$-semilattices can be lifted by a positive homomorphism between countable dimension vector spaces, cannot be extended to uncountable semilattices:

Example 11.1. Let $B=\left\{x \subseteq \omega_{1} \mid\right.$ either $x$ or $\omega_{1} \backslash x$ is finite $\}$. So $B$ is a Boolean semilattice of size $\aleph_{1}$. Let $\boldsymbol{s}: B \rightarrow \mathbf{2}$ be the simplest closure operator on $B$, that is, $\boldsymbol{s}(0)=0$ while $\boldsymbol{s}(\boldsymbol{x})=1$, for all $\boldsymbol{x} \in B \backslash\{0\}$. Then $\boldsymbol{s}$ has no lifting, that is, there are no positive homomorphism $s: G \rightarrow S$, for some partially ordered abelian groups $G$ and $S$ such that $G$ has interpolation, and no isomorphisms $\alpha: B \rightarrow \operatorname{Id}_{\mathrm{c}} G$ and $\varepsilon: \mathbf{2} \rightarrow \operatorname{Id}_{\mathrm{c}} S$ such that the following diagram is commutative:

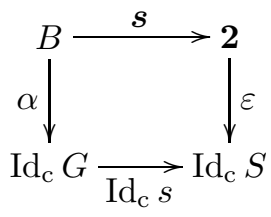


Proof. Suppose otherwise. In particular, $S$ is a simple partially ordered abelian group. Let $\alpha\left(1_{B}\right)=G(u)$, for some $u \in G^{++}$. For all $\xi<\omega_{1}$, the inequality $\{\xi\}<$ $1_{B}$ holds, so $\alpha(\{\xi\})<\alpha\left(1_{B}\right)=G(u)$, thus there are $m_{\xi} \in \mathbb{N}$ and $u_{\xi} \in\left[0, m_{\xi} u\right]$ in $G$ such that $G\left(u_{\xi}\right)=\alpha(\{\xi\})$. There exists an uncountable subset $U$ of $\omega_{1}$ such that $m_{\xi}=m=$ constant, for all $\xi \in U$, therefore we may replace $u$ by $m u$ and assume that $0 \leq u_{\xi} \leq u$, for all $\xi \in U$, thus there exists $v_{\xi} \in[0, u]$ such that $u_{\xi}+v_{\xi}=u$. Furthermore, for $\xi \neq \eta$ in $\omega_{1},\{\xi\} \cap\{\eta\}=\emptyset$, thus $G\left(u_{\xi}\right) \cap G\left(u_{\eta}\right)=\{0\}$, hence $u_{\xi} \wedge u_{\eta}=0$ in $G$.

By applying this last fact together with Lemma 3.1] in the refinement monoid $G^{+}$ (with $I=A$ and $T_{\xi}=\{0,1\}$, for all $\xi \in A$ ) to the system of equalities $u_{\xi}+v_{\xi}=u$ (for all $\xi \in A$ ), we obtain the inequality

$$
\sum_{\xi \in A} u_{\xi} \leq u, \quad \text { for all finite } A \subset U .
$$

Next, since $s$ lifts $s, s\left(u_{\xi}\right) \in S^{++}$, for all $\xi \in U$, thus, since $S$ is simple, there exists $n_{\xi} \in \mathbb{N}$ such that $s(u) \leq n_{\xi} s\left(u_{\xi}\right)$. Let $V \subseteq U$ uncountable such that $n_{\xi}=n=$ constant, for all $\xi \in V$. Pick any distinct $\xi_{0}, \ldots, \xi_{n} \in V$. For all $i \in\{0, \ldots, n\}$, the inequalities $s(u) \leq n_{\xi_{i}} \cdot s\left(u_{\xi_{i}}\right)=n \cdot s\left(u_{\xi_{i}}\right)$ hold, hence, adding together all these inequalities, we obtain that

$$
\begin{aligned}
(n+1) \cdot s(u) & \leq n \cdot \sum_{i=0}^{n} s\left(u_{\xi_{i}}\right) \\
& =n \cdot s\left(\sum_{i=0}^{n} u_{\xi_{i}}\right) \\
& \leq n \cdot s(u) \quad \text { (by (11.1) }),
\end{aligned}
$$

whence $s(u) \leq 0$, a contradiction.

\section{Open PROBLEMS}

We first recall the following central open problem, which is the basic motivation of the present paper:

Problem 1 (Problem 10.1 of [8]). Let $S$ be a $\langle\vee, 0\rangle$-semilattice of cardinality $\aleph_{1}$. Does there exist a dimension group $G$ such that $\operatorname{Id}_{\mathrm{c}} G \cong S$ ?

A related problem is the following.

Problem 2. Can every finite diagram (indexed by a non necessarily dismantlable partially ordered set) of finite Boolean semilattices and $\langle\vee, 0\rangle$-homomorphisms be lifted by a diagram of dimension groups and positive homomorphisms?

Example 9.1 shows that the analogue of Problem 2 for diagrams indexed by arbitrary finite categories (instead of partially ordered sets) fails. Still the problem for an arbitrary finite partially ordered index set $P$ remains, in particular for $P=$ $2^{3}$.

We do not know about the ring-theoretical analogue of Theorem 6.4

Problem 3. Let $K$ be a field. Is it the case that every diagram of finite Boolean $\langle\vee, 0\rangle$-semilattices that is indexed by a finite dismantlable partially ordered set can be lifted, with respect to the $\operatorname{Id}_{\mathrm{c}}$ functor on rings, by a diagram of locally matricial algebras over $K$ ? 
We do not even know whether Problem 3 has a positive solution for the square diagram of Example10.1 On the other hand, it follows from the results of F. Wehrung [19] that every square diagram of finite Boolean semilattices can be lifted, with respect to the $\mathrm{Id}_{\mathrm{c}}$ functor, by a diagram of von Neumann regular algebras (over any given field).

Problem 4. Let $G$ be a dimension group, let $S$ be a distributive $\langle\vee, 0\rangle$-semilattice, and let $\boldsymbol{f}: \operatorname{Id}_{\mathrm{c}} G \rightarrow S$ be a $\langle\vee, 0\rangle$-homomorphism. Find sufficient conditions under

which $f$ can be lifted, that is, there are a dimension group $H$ and an isomorphism $\beta: S \rightarrow \operatorname{Id}_{\mathrm{c}} H$ such that $\operatorname{Id}_{\mathrm{c}} f=\beta \circ \boldsymbol{f}$ ?

Example 8.1 shows that strong restrictions on $G, S$, and $f$ are needed. Compare also with Theorem 7.1

\section{ACKNOWLEDGMENTS}

Part of this work was completed while the second author was visiting the department of mathematics at Charles University in Prague while staying at the Suchdol campus. The excellent conditions provided by both places are greatly appreciated. In particular, special thanks are due to Václav Slavík.

Another part of the work was completed while the first author visited the University of Caen supported by the Barrande Program. Special thanks also to Philippe Toffin.

\section{REFERENCES}

[1] K. A. Baker, P. C. Fishburn, and F. S. Roberts, Partial orders of dimension 2, interval orders, and interval graphs, Rand Corp. P-4376, 1970.

[2] G. M. Bergman, Von Neumann regular rings with tailor-made ideal lattices, Unpublished notes (26 October 1986).

[3] E. G. Effros, D. E. Handelman, and C.-L. Shen, Dimension groups and their affine representations, Amer. J. Math. 102 (1980), no. 2, 385-407.

[4] G. A. Elliott, On the classification of inductive limits of sequences of semisimple finitedimensional algebras, J. Algebra 38 (1976), 29-44.

[5] K. R. Goodearl, "Von Neumann Regular Rings", Pitman, London 1979; Second Ed. Krieger, Malabar, Fl., 1991.

[6] _ "Partially Ordered Abelian Groups with Interpolation", Math. Surveys and Monographs 20, Amer. Math. Soc., Providence, 1986.

[7] K. R. Goodearl and D. E. Handelman, Tensor products of dimension groups and $K_{0}$ of unitregular rings, Canad. J. Math. 38, no. 3 (1986), 633-658.

[8] K. R. Goodearl and F. Wehrung, Representations of distributive semilattices in ideal lattices of various algebraic structures, Algebra Universalis 45 (2001), 71-102.

[9] G. Grätzer, "General Lattice Theory. Second edition", new appendices by the author with B. A. Davey, R. Freese, B. Ganter, M. Greferath, P. Jipsen, H. A. Priestley, H. Rose, E. T. Schmidt, S. E. Schmidt, F. Wehrung, and R. Wille. Birkhäuser Verlag, Basel, 1998. xx+663 p.

[10] A.P. Huhn, On the representation problem of distributive algebraic lattices, III, Acta Sci. Math. (Szeged) 53 (1989), 11-18.

[11] D. Kelly and I. Rival, Crowns, fences, and dismantlable lattices, Canad. J. Math. 26 (1974), $1257-1271$.

[12] _ Planar lattices, Canad. J. Math. 27 (1975), 636-665.

[13] P. Pudlák, On congruence lattices of lattices, Algebra Universalis 20 (1985), 96-114.

[14] P. Růžička, A distributive semilattice not isomorphic to the maximal semilattice quotient of the positive cone of any dimension group, J. Algebra, to appear.

[15] A. Tarski, "Cardinal Algebras", New York: Oxford, 1949.

[16] F. Wehrung, Injective positively ordered monoids I, J. Pure Appl. Algebra 83 (1992), 43-82. 
[17] _ Non-measurability properties of interpolation vector spaces, Israel J. Math. 103 (1998), 177-206.

[18] - A uniform refinement property for congruence lattices, Proc. Amer. Math. Soc. 127, no. 2 (1999), 363-370.

[19] Representation of algebraic distributive lattices with $\aleph_{1}$ compact elements as ideal lattices of regular rings, Publ. Mat. 44 (2000), no. 2, 419-435.

Department of Algebra, Faculty of Mathematics and Physics, Sokolovská 83, Charles University, 18600 Praha 8, Czech Republic

E-mail address: tuma@karlin.mff.cuni.cz

CNRS, UMR 6139, Université de Caen, Campus II, Département de Mathématiques, B.P. 5186, 14032 CAEN CEDEX, FRANCE

E-mail address: wehrung@math.unicaen.fr

$U R L$ : http://www.math.unicaen.fr/ wehrung 